\title{
Short Wavelength Cutoff Effects in the AC Fluctuation Conductivity of Superconductors
}

\author{
D.-N. Peligrad and M. Mehring \\ 2. Physikalisches Institut, Universität Stuttgart, 70550 Stuttgart, Germany
}

\author{
A. Dulčić \\ Department of Physics, Faculty of Science, University of Zagreb, POB 331, 10002 Zagreb, Croatid*
}

(Dated: Submitted February 28, 2002; revised version November 26, 2002)

\begin{abstract}
The short wavelength cutoff has been introduced in the calculation of $a c$ fluctuation conductivity of superconductors. It is shown that a finite cutoff leads to a breakdown of the scaling property in frequency and temperature. Also, it increases the phase $\phi$ of the complex conductivity $\left(\tan \phi=\sigma_{2} / \sigma_{1}\right)$ beyond $\pi / 4$ at $T_{c}$. Detailed expressions containing all essential parameters are derived for $3 \mathrm{D}$ isotropic and anisotropic fluctuation conductivity. In the $2 D$ case we obtain individual expressions for the fluctuation conductivity for each term in the sum over discrete wavevectors perpendicular to the film plane. A comparison of the theory to the experimental microwave fluctuation conductivity is provided.
\end{abstract}

PACS numbers: 74.40.+k, 74.25.Nf, 74.76.Bz

\section{INTRODUCTION}

Fluctuations of the order parameter near the critical temperature $T_{c}$ are much larger in high- $T_{c}$ superconductors than in classical low temperature superconductors. One of the reasons lies in the higher thermal energy $k_{\mathrm{B}} T_{c}$ which provides the excitations, and the other in a very short coherence lengths which occur in high- $T_{c}$ cuprate superconductors. With these properties, the region of critical fluctuations was estimated from the Ginzburg criterion to be of the order of $1 \mathrm{~K}$, or more, around $T_{c}$, which renders the critical region accessible to experimental investigations $\stackrel{1}{\stackrel{1}{*} \text { Farther above }}$ $T_{c}$, one expects to observe the transition from critical to noninteracting Gaussian fluctuations which are the lowest order fluctuation corrections to the mean field theory ${ }^{2}$

The layered structure of high- $T_{c}$ superconductors requires some theoretical sophistication. One could treat these superconductors with various models from three-dimensional (3D) anisotropic to coupled layers Lawrence-Doniach, or purely two-dimensional (2D) ones. Due to the temperature variation of the coherence lengths one could even expect a dimensional crossover in some systems. The fluctuation conductivity is altered by dimensionality in various models so that a detailed comparison of model calculations and experimental data could address the dimensionality problem.

For the reasons stated above, the fluctuation conductivity in high- $T_{c}$ superconductors was studied experimentally

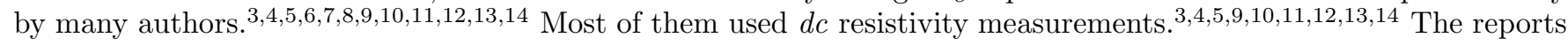
were controversial in the conclusions about the dimensionality of the system, and the critical exponents. It has been shown that, in a wide temperature range above $T_{c}$, the fluctuation conductivity did not follow any of the single exponent power laws predicted by scaling and mean-field theories $\stackrel{5}{\underline{5}}$ The data in the Gaussian regime could be fitted by an expression derived within the Ginzburg-Landau (GL) theory with a short wavelength cutoff in the fluctuation spectrum. Recently, Silva et al $\stackrel{13}{\underline{n}}$ have proven that the GL approach with an appropriate choice of the cutoff parameter yields result which is identical to that of the microscopic Aslamazov-Larkin (AL) approach with reduced excitations of the short wavelength fluctuations $\frac{15}{\underline{15}}$ It has been further shown that the detailed temperature dependence of the fluctuation conductivity was not universal, but sample dependent. In this respect, the GL approach has practical advantage since the cutoff parameter can be readily adjusted in fitting the experimental data. Silva et al $\stackrel{13}{\underline{\underline{n}}}$ could fit very well the data on a number of thin films in the Gaussian region from $T_{c}+1 \mathrm{~K}$ to $T_{c}+25 \mathrm{~K}$.

When critical fluctuations are studied, it becomes essential to know accurately the value of $T_{c}$. However, the determination of $T_{c}$ from $d c$ resistivity measurements brings about some uncertainties. One should avoid the use of unjustifiable definitions of $T_{c}$ such as e. g.: (i) zero resistance temperature, (ii) midpoint of the transition, (iii) maximum of the derivative $d \rho / d T$, (iv) intersection of the tangent to the transition curve with the temperature axis, etc. The correct value of the critical temperature can be determined as an additional fitting parameter in the analysis of the fluctuation conductivity. Usually one assumes that a well defined power law holds in a given narrow temperature range and then determine both, $T_{c}$ and the critical exponent from the selected segment $10.12,14$ However, the experimental data usually show an almost continuous change of the slope so that the uncertainty in the determination of $T_{c}$ is an unsolved problem. Besides, the effects of the cutoff have been neglected in the analysis of the data close to $T_{c}$. Even though the values of the fluctuation conductivity near $T_{c}$ are not much affected by the

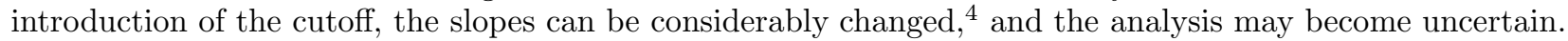


A number of microwave studies have been reported showing clear signs of fluctuations in both, the real and imaginary parts of the $a c$ conductivity $\underline{6.7 .16 .17 .18}$ The real part $\sigma_{1}$ of the complex conductivity $\left(\widetilde{\sigma}=\sigma_{1}-i \sigma_{2}\right)$ has a sharp peak at $T_{c}$, which is not observed in e.g. $\mathrm{Nb}$ as a representative of low temperature classical superconductors ${ }^{19}$ The salient feature of the $a c$ case is that the fluctuation conductivity does not diverge at $T_{c}$ because a finite frequency provides a limit to the observation of the critical slowing down near $T_{c}$. The real part $\sigma_{1}$ has a maximum at $T_{c}$. It is also important to note that $\sigma_{1}$ and $\sigma_{2}$ have individually different temperature and frequency dependences, even though they result from the same underlying physics. Testing a given theoretical model becomes more stringent when two curves have to be fitted with the same set of parameters.

The expressions for the $a c$ fluctuation conductivity in the Gaussian regime have been deduced within the time dependent Ginzburg-Landau (TDGL) theory by Schmidt. ${ }^{20}$ Using general physical arguments, Fisher, Fisher, and Huse $^{21}$ provided a formulation for the scaling of the complex $a c$ conductivity as

$$
\widetilde{\sigma}(\omega) \propto \xi^{z+2-D} \widetilde{\mathcal{S}}_{ \pm}\left(\omega \xi^{z}\right)
$$

where $\xi$ is the correlation length, $z$ is the dynamical critical exponent, $D$ is the dimensionality of the system, and $\widetilde{\mathcal{S}}_{ \pm}\left(\omega \xi^{z}\right)$ are some complex scaling functions above and below $T_{c}$. This form of the fluctuation conductivity was claimed to hold in both, the Gaussian and critical regimes. Dorsey ${ }^{22}$ has deduced the scaling functions in the Gaussian regime above $T_{c}$, and verified the previous results of Schmidt $\underline{\underline{20}}$ More recently, Wickham and Dorsey $\underline{23}$ have shown that even in the critical regime, where the quartic term in the GL free energy plays a role, the scaling functions preserve the same form as in the Gaussian regime.

The above mentioned theoretical expressions of the $a c$ fluctuation conductivity did not take into account the slow variation approximation which is required for the validity of the Ginzburg-Landau theory ${ }^{2} \underline{\underline{2}}$ It was noted long time ago that the summation over the fluctuation modes had to be truncated at a wavevector which corresponded roughly to the inverse of the intrinsic coherence length $\xi_{0} \underline{24}$ The improved treatment with a short wavelegth cutoff was applied in fluctuation diamagnetism,${ }^{25}$ and $d c$ paraconductivity far above $T_{c}{ }^{26}$ This approach was also applied in $d c$ fluctuation conductivity of high- $T_{c}$ superconductors where one encounters a large anisotropy ${ }^{4.5 .13}$ The introduction of the short wavelength cutoff was found to be essential in fitting the theoretical expressions to the experimental data. In view of the great potential of the microwave method described above, we find motivation to elaborate in this paper the improved theory of $a c$ fluctuation conductivity including the short wavelength cutoff. We find that the resulting expressions can be written in the form of Eq. (11). However, the cutoff introduces a breakdown of the scaling property in the variable $\omega \xi^{z}$. Also, we find that the phase $\phi$ of the complex conductivity $\left(\tan \phi=\sigma_{2} / \sigma_{1}\right)$ evaluated at $T_{c}$ departs from the value $\pi / 4$ when cutoff is introduced. Values of $\phi$ larger than $\pi / 4$ were observed experimentally, ${ }^{7}$ but were attributed to an unusually large dynamic critical exponent. Also, deviation of the scaling in the variable $\omega \xi^{z}$ was observed already at $2 \mathrm{~K}$ above $T_{c}, \frac{7}{\underline{7}}$ but no analysis was made considering the short wavelength cutoff in the fluctuation spectrum. The present theory is developed for different dimensionalities which facilitates comparison with experimental data.

\section{THE EFFECTS OF SHORT WAVELENGTH CUTOFF}

Frequency dependent conductivity can be calculated within the Kubo formalism from the current correlation function. For the fluctuation conductivity one has to consider the current due to the fluctuations of the order parameter. The resulting expression for the real part of the conductivity is 27

$$
\sigma_{1}^{x x}=\left(\frac{2 e \hbar}{m}\right)^{2} \frac{1}{k_{B} T} \sum_{\mathbf{k}} k_{x}^{2}<\left|\psi_{\mathbf{k}}\right|^{2}>^{2} \frac{\tau_{k} / 2}{1+\left(\omega \tau_{k} / 2\right)^{2}},
$$

where the current is assumed to be in the $x$-direction. $\psi_{k}$ is the Fourier component of the order parameter, and $\tau_{k}=\tau_{0} /\left(1+\xi^{2} k^{2}\right)$ is the relaxation time of the $k$-th component. The relaxation time for the $k=0$ mode is given by

$$
\tau_{0}=\frac{\pi \hbar}{8 k_{B} T_{c}}\left(\frac{\xi(T)}{\xi_{0}}\right)^{z}
$$

where $z$ is the dynamic critical exponent. An alternative approach is to calculate the response of the system to an external field through the expectation value of the current operator averaged with respect to the noise ${ }^{22}$ However, the introduction of the short wavelength cutoff in this approach leads only to selfconsistent implicit expressions ${ }^{28.29}$

Eq. (21) is obtained from the time dependent Ginzburg-Landau theory and represents the equivalent of the Aslamazov-Larkin fluctuation conductivity obtained from microscopic calculations. In the following, we present the results which take account of the short wavelength cutoff in this contribution to the ac fluctuation conductivity. The 
other contributions such as Maki-Thomson (MT) and one-electron density of states (DOS) renormalization 30.31 .32 cannot be treated within the time dependent Ginzburg-Landau theory but require microscopic calculations. It has been shown ${ }^{31}$ that MT anomalous contribution in high- $T_{c}$ superconductors is almost temperature independent while DOS contribution is strongly temperature dependent, and contains a number of parameters which have to be determined through a complex fitting procedure in an experimental data analysis ${ }^{33}$ Since the three terms in the fluctuation conductivity are additive it is important to have the Aslamazov-Larkin term corrected for short wavelength cutoff which then allows to fit the MT and DOS contributions properly from the rest of the total experimental fluctuation conductivity.

The sum in Eq. (2) can be evaluated by integration considering the appropriate dimensionality. In this section we discuss the simplest case of an isotropic $3 D$ superconductor. The integration in $\mathbf{k}$-space needs a cutoff since the order parameter cannot vary appreciably over distances which are shorter than some minimum wavelength. The cutoff in $k_{x}$ can be expressed as $k_{x}^{\text {max }}=\Lambda / \xi_{0}$, where $\Lambda$ is a dimensionless cutoff parameter. Obviously, $\Lambda \rightarrow \infty$ would imply no cutoff in the integration, whereas for $\Lambda \approx 1$ one obtains the usually assumed cutoff at $1 / \xi_{0}$. In the $3 D$ isotropic case, the same cutoff applies also to $k_{y}$ and $k_{z}$ so that for the $3 D$ integration in the $\mathbf{k}$-space one has to set the cutoff limit for the modulus $k^{\max }=\sqrt{3} \Lambda / \xi_{0}$. With the change of variable $q(T)=k \xi(T)$ one obtains

$$
\sigma_{1}^{3 D, i s o}(\omega, T, \Lambda)=\frac{e^{2}}{6 \pi \hbar \xi_{0}}\left(\frac{\xi(T)}{\xi_{0}}\right)^{z-1} \int_{0}^{Q} \frac{q^{4}}{\left(1+q^{2}\right)\left[\Omega^{2}+\left(1+q^{2}\right)^{2}\right]} d q
$$

where

$$
Q(T, \Lambda)=k_{\text {cut }} \xi(T)=\sqrt{3} \Lambda\left(\frac{\xi(T)}{\xi_{0}}\right)
$$

is the temperature dependent cutoff limit in the $q$-space, and

$$
\Omega(\omega, T)=\frac{\omega \tau_{0}}{2}=\frac{\pi}{16} \frac{\hbar \omega}{k_{B} T_{c}}\left(\frac{\xi(T)}{\xi_{0}}\right)^{z}
$$

is a dimensionless variable which depends on frequency and temperature as independent experimental variables.

For the $d c$ case $(\omega=0)$, and no cutoff $(\Lambda \rightarrow \infty)$, one finds from Eq. (4)

$$
\sigma_{d c}^{3 D, i s o}(T, \Lambda \rightarrow \infty)=\frac{e^{2}}{32 \hbar \xi_{0}}\left(\frac{\xi(T)}{\xi_{0}}\right)^{z-1}
$$

which reduces to the well known Aslamazov-Larkin result $\frac{27}{47}$ provided that relaxational dynamics is assumed $(z=2)$, and $\xi(T) / \xi_{0}$ is taken only in the Gaussian limit as $1 / \sqrt{\epsilon}$. However, with a finite cutoff parameter $\Lambda$ one obtains

$$
\sigma_{d c}^{3 D, i s o}(T, \Lambda)=\frac{e^{2}}{16 \pi \hbar \xi_{0}}\left(\frac{\xi(T)}{\xi_{0}}\right)^{z-1}\left[\arctan (Q)-Q \frac{\left(\frac{5}{3} Q^{2}+1\right)}{\left(1+Q^{2}\right)^{2}}\right] .
$$

This result has been obtained by Hopfengärtner et. al $\stackrel{4}{*}$ except that they used only the Gaussian limit $1 / \sqrt{\epsilon}$ for the reduced correlation length $\xi(T) / \xi_{0}$. Their analysis has shown that the cutoff plays no role exactly at $T_{c}$ since $Q \rightarrow \infty$ regardless of $\Lambda$. However, at any temperature above $T_{c}$ one gets a finite $Q$ and the value of the conductivity is lowered with respect to the result given by Eq. (7). Their conclusion was that the Gaussian fluctuations with no cutoff yield an overestimated fluctuation conductivity.

In this paper we are primarily interested in the ac case. Before integrating Eq. (4) with $\Omega \neq 0$, we find the corresponding expression for the imaginary part $\sigma_{2}$. We can apply Kramers-Kronig relations to each of the Fourier components in Eq. (2), and carry out the summation. This is equivalent to a calculation of the kernel $\mathcal{K}_{2}$ for $\sigma_{2}$ from the kernel $\mathcal{K}_{1}$ used in Eq. (4), namely

$$
\mathcal{K}_{2}(\Omega)=\frac{2 \Omega}{\pi} \int_{0}^{\infty} \frac{\mathcal{K}_{1}\left(\Omega^{\prime}\right)}{\Omega^{2}-\Omega^{\prime 2}} d \Omega^{\prime}
$$

With the kernel $\mathcal{K}_{2}(\Omega)$, the imaginary part of the fluctuation conductivity can be calculated for any cutoff parameter $\Lambda$

$$
\sigma_{2}^{3 D, i s o}(\omega, T, \Lambda)=\frac{e^{2}}{6 \pi \hbar \xi_{0}}\left(\frac{\xi(T)}{\xi_{0}}\right)^{z-1} \int_{0}^{Q} \frac{\Omega q^{4}}{\left(1+q^{2}\right)^{2}\left[\Omega^{2}+\left(1+q^{2}\right)^{2}\right]} d q
$$


Finally, the complex fluctuation conductivity can be written in the form

$$
\widetilde{\sigma}^{3 D, i s o}(\omega, T, \Lambda)=\frac{e^{2}}{32 \hbar \xi_{0}}\left(\frac{\xi(T)}{\xi_{0}}\right)^{z-1}\left[\mathcal{S}_{1}^{3 D, i s o}(\omega, T, \Lambda)+i \mathcal{S}_{2}^{3 D, i s o}(\omega, T, \Lambda)\right] .
$$

The prefactor is equal to the $d c$ result with no cutoff effect as in Eq. (7). The functions $\mathcal{S}_{1,2}$ are given by the following expressions

$$
\begin{gathered}
\mathcal{S}_{1}^{3 D, i s o}(\omega, T, \Lambda)=\frac{1}{3 \pi \Omega^{2}}\left[P_{-}\left(P_{+}^{2}+2\right) L+2 P_{+}\left(P_{-}^{2}-2\right) A+16 \arctan (Q)\right], \\
\mathcal{S}_{2}^{3 D, i s o}(\omega, T, \Lambda)=\frac{1}{3 \pi \Omega^{2}}\left[2 P_{-}\left(P_{+}^{2}+2\right) A-P_{+}\left(P_{-}^{2}-2\right) L-24 \Omega \arctan (Q)+8 \Omega \frac{Q}{1+Q^{2}}\right],
\end{gathered}
$$

where we used the following shorthand notations

$$
\begin{gathered}
P_{ \pm}=\sqrt{2} \sqrt{\sqrt{\Omega^{2}+1} \pm 1} \\
L=\ln \left(\frac{2+Q^{2}+\left(Q-P_{-}\right)^{2}}{2+Q^{2}+\left(Q+P_{-}\right)^{2}}\right), \\
A=\arctan \left(\frac{2 Q+P_{-}}{P_{+}}\right)+\arctan \left(\frac{2 Q-P_{-}}{P_{+}}\right) .
\end{gathered}
$$

It can be easily verified that the $\mathcal{S}_{1,2}$-functions given by Eq. (12) and Eq. (13) have proper limits. In the $d c$ limit $(\Omega \rightarrow 0)$, one finds that $\mathcal{S}_{2} \rightarrow 0$, and $\mathcal{S}_{1}$ leads to the $d c$ result of Eq. (8). One can also verify that the $a c$ results obtained previously by Schmidt ${ }^{20}$ and Dorsey ${ }^{22}$ can be recovered from our Eq. (12) and Eq. (13) in the limit $\Lambda \rightarrow \infty$, i. e. when no cutoff is made.

The effects of the cutoff are not trivial in the $a c$ case. It is essential to examine those effects in detail as they have strong bearing on the analysis of the experimental data. The prefactor in Eq. (11) depends only on temperature while the cutoff parameter $\Lambda$ is found only in the $\mathcal{S}$-functions. Therefore, the effects of the cutoff can be studied through the $\mathcal{S}$-functions alone. We can look at the temperature and frequency dependences of these functions with and without

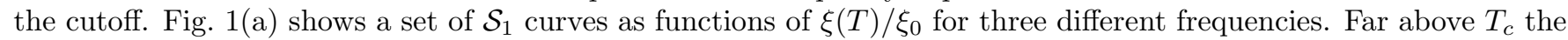
relaxation time $\tau_{0}$ is so short that $\omega \tau_{0} \ll 1$ for any of the chosen frequencies. Therefore the response of the system is like in the $d c$ case. With no cutoff, $\mathcal{S}_{1}$ saturates to unity (dashed lines in Fig. 1(a)). This limit is required in order that $\sigma_{1}$ from Eq. (11) becomes equal to $\sigma_{d c}(\Lambda \rightarrow \infty)$ in Eq. (7). If a cutoff with a finite $\Lambda$ is included, $\mathcal{S}_{1}$ decays at higher temperatures (solid lines in Fig. 团(a)). The reduction of $\mathcal{S}_{1}$ is more pronounced at smaller values of $\xi(T)$ since the integration in the $q$-space is terminated at a lower value $Q=\sqrt{3} \Lambda \xi(T) / \xi_{0}$. At higher temperatures the conductivity $\sigma_{1}$ at any frequency behaves asymptotically as $\sigma_{d c}$ given by Eq. (8).

At temperatures closer to $T_{c}$ the relaxation time $\tau_{0}$ increases as $\xi(T)^{z}$ with increasing correlation length according to Eq. (3) which is usually termed critical slowing down. When $\omega \tau_{0} \approx 1$ for a given frequency, $\mathcal{S}_{1}$ is sharply reduced and vanishes in the limit of $T_{c}$. With the diverging prefactor in Eq. (11) it can still yield a finite $\sigma_{1}$ at $T_{c}$. It may appear from Fig. I(a) that cutoff makes no effect when $T_{c}$ is approached, but we show later that an important feature still persists in $\sigma_{1}$.

Obviously, at lower operating frequencies one needs to approach $T_{c}$ closer so that the critical slowing down could reach the condition $\omega \tau_{0} \approx 1$. One can see from Fig. 目a) that for frequencies below $1 \mathrm{GHz}$ one would have to approach $T_{c}$ closer than $1 \mathrm{mK}$ in order to probe the critical slowing down in fluctuations. The higher the frequency, the farther above $T_{c}$ is the temperature where the crossover $\omega \tau_{0} \approx 1$ occurs. This feature expresses the scaling property of the conductivity in frequency and temperature variables. However, the scaling property holds strictly only in the absence of the cutoff. Namely, if one sets $\Lambda \rightarrow \infty$, the function $\mathcal{S}_{1}$ depends only on the scaling variable $\Omega$. Fig. 1(b) shows the same set of curves as in Fig. 1(a), but plotted versus $\Omega$. The three dashed curves from Fig. 1(a) coalesce into one dashed curve in Fig. I(b), thus showing the scaling property in the absence of cutoff. However, the full lines representing the functions $\mathcal{S}_{1}$ with a finite cutoff parameter $\Lambda$ do not scale with the variable $\Omega$. The reason is that the function $\mathcal{S}_{1}$ then depends also on $Q$, which itself is not a function of $\Omega$. Namely, the cutoff in the $q$-space depends on the properties of the sample, and on the temperature, but not on the frequency used in the experiment. Hence, the 
cutoff brings about a breakdown of the scaling property in frequency and temperature. The effect is more pronounced at temperatures farther above $T_{c}$ where the cutoff is stronger.

The properties of the function $\mathcal{S}_{2}$ are shown in Fig. 2 for the same set of three measurement frequencies as in Fig. 1 . When plotted versus $\xi(T) / \xi_{0}$, the function $\mathcal{S}_{2}$ exhibits a maximum at the point where the corresponding function $\mathcal{S}_{1}$ shows the characteristic crossover due to $\omega \tau_{0} \approx 1$ as discussed above. When $T_{c}$ is approached, $\mathcal{S}_{2}$ tends to zero. When $\mathcal{S}_{2}$ is multiplied with the diverging prefactor in Eq. (11), one finds a finite $\sigma_{2}$ at $T_{c}$. Far above $T_{c}$, the function $\mathcal{S}_{2}$ vanishes, regardless of the cutoff. This is consistent with the behavior of $\mathcal{S}_{1}$. Namely, at high enough temperatures, $\mathcal{S}_{1}$ acquires asymptotically the $d c$ value, as seen in Fig. 1 Obviously, the imaginary part of the conductivity must vanish when $d c$ like limit is approached. The decrease of the function $\mathcal{S}_{2}$ at higher temperatures is very rapid so that the effects of the cutoff are unnoticeable on the linear scale. Only with the logarithmic scale used in the inset to Fig. 2(a), one observes that the cutoff effects are present also in $\mathcal{S}_{2}$, though by a very small amount. Fig. 22(b) shows the scaling property of $\mathcal{S}_{2}$ with no cutoff and its breakdown when cutoff is included.

We have noted above that both, $\mathcal{S}_{1}$ and $\mathcal{S}_{2}$ tend to zero when $T_{c}$ is approached. Also, the effect of cutoff is seen to be small in that limit. Yet, these functions are multiplied by the diverging prefactor in Eq. (11), and then may yield finite $\sigma_{1}$ and $\sigma_{2}$. A careful analysis is needed in order to find the phase $\phi$ of the complex conductivity $\left(\phi=\arctan \left(\sigma_{2} / \sigma_{1}\right)\right)$ at $T_{c}$. For a $3 D$ isotropic superconductor, Dorsey ${ }^{22}$ has predicted $\phi=\pi / 4$, i. e. $\sigma_{1}=\sigma_{2}$ at $T_{c}$. His result was obtained with no cutoff and it remains to be seen if this property is preserved even when a finite cutoff is made. Fig. 31(a) shows $\mathcal{S}_{1}$ and $\mathcal{S}_{2}$ as functions of $\xi(T) / \xi_{0}$ for $100 \mathrm{GHz}$ frequency. The effects of cutoff on $\mathcal{S}_{2}$ are noticeable only far above $T_{c}$. Closer to $T_{c}$, the curves for $\mathcal{S}_{2}$ calculated with, and without cutoff, are indistinguishable. In contrast, a finite cutoff reduces the values of $\mathcal{S}_{1}$ even in the limit of $T_{c}$. As a result, the final cutoff parameter $\Lambda$ yields a crossing of the curves for $\mathcal{S}_{1}$ and $\mathcal{S}_{2}$ at some temperature slightly above $T_{c}$. It is better seen on an enlarged scale in Fig. [3 b). This is a surprising result which has bearing on the experimental observations. Due to the cutoff, the condition $\mathcal{S}_{1}=\mathcal{S}_{2}$ $(\phi=\pi / 4)$ is reached at a temperature slightly above $T_{c}$. Since both, $\mathcal{S}_{1}$ and $\mathcal{S}_{2}$ are multiplied with the same prefactor in Eq. (11), one finds that the crossing of $\sigma_{1}(T)$ and $\sigma_{2}(T)$ does not occur at the peak of $\sigma_{1}(T)$, but at a slightly higher temperature. Exactly at $T_{c}, \sigma_{2}$ is higher than $\sigma_{1}$ since a finite cutoff parameter reduces $\sigma_{1}$, but makes no effect on $\sigma_{2}$.

The observation that the cutoff brings about a reduction of $\sigma_{1}$ at $T_{c}$ is worth further investigation since it can be measured experimentally. Fig. 4 (a) shows the ratio $\mathcal{S}_{2} / \mathcal{S}_{1}$ (equal to $\sigma_{2} / \sigma_{1}$ ) at temperatures approaching $T_{c}$. With no cutoff (dashed lines in Fig. 4l(a)), this ratio reaches unity regardless of the frequency used. A finite cutoff parameter $\left(\Lambda=0.5\right.$ in Fig. 4(a)) makes the ratio equal to unity at a temperature slightly above $T_{c}$, and in the limit of $T_{c}$ the ratio saturates at some higher value. The saturation level is seen to be higher when a higher frequency is used.

One can find analytical expansions of the $\mathcal{S}$-functions in the limit of $T_{c}(\Omega \rightarrow \infty)$. The leading terms are

$$
\mathcal{S}_{1,2}^{3 D, i s o}(W, \Omega \rightarrow \infty) \approx \frac{4 \sqrt{2}}{3 \pi}\left(C \mp \frac{1}{2} D\right) \frac{1}{\sqrt{\Omega}}
$$

where we used the notation

$$
\begin{gathered}
C=\arctan (1+\sqrt{2} W)-\arctan (1-\sqrt{2} W), \\
D=\ln \left(\frac{1+\sqrt{2} W+W^{2}}{1-\sqrt{2} W+W^{2}}\right) .
\end{gathered}
$$

The parameter $W$ depends on the frequency $\omega$ and the cutoff parameter $\Lambda$

$$
W=\sqrt{3} \Lambda \sqrt{\frac{16}{\pi} \frac{k_{B} T_{c}}{\hbar \omega}} .
$$

Both functions tend to zero in the limit of $T_{c}(\Omega \rightarrow \infty)$, but their ratio is finite and depends on the parameter $W$. Fig. 4(b) shows the plot of $\mathcal{S}_{2} / \mathcal{S}_{1}$ at $T_{c}$ as the function of $W$. One can observe that for a given cutoff parameter $\Lambda$, the ratio $\mathcal{S}_{2} / \mathcal{S}_{1}$ at $T_{c}$ increases at higher frequencies (lower $W$ ). The limits at $T_{c}$ in Fig. 4 (a) represent only three selected points on the curve for $\mathcal{S}_{2} / \mathcal{S}_{1}$ in Fig. 4 (b).

In a given experiment, the ratio $\sigma_{2} / \sigma_{1}$ at $T_{c}$ can be directly determined from the experimental data so that the corresponding value of the parameter $W$ can be found uniquely from the curve of $\mathcal{S}_{2} / \mathcal{S}_{1}$ in Fig. 远(b), and the cutoff parameter $\Lambda$ is obtained using Eq. (20). We should note that $\Lambda$ is a temperature independent parameter. It can be determined by the above procedure from the experimental data at $T_{c}$, but it controls the cutoff at all temperatures.

One may observe from Eq. (17) that in the limit of $T_{c}$ the leading terms in the expansions of the $\mathcal{S}$-functions behave as $\left(\xi(T) / \xi_{0}\right)^{-z / 2}$. Taking into account the prefactor in Eq. (11) one finds that $\sigma_{1}$ and $\sigma_{2}$ can have finite nonzero 
values at $T_{c}$ only if $z=2$, i. e. for the purely relaxational dynamics. We have assumed this case in all the figures of this section.

From the experimental data at $T_{c}$ one can determine also the parameter $\xi_{0}$. Using Eq. (11) and the $\mathcal{S}$-functions in Eq. (17), one obtains finite conductivities at $T_{c}$

$$
\sigma_{1,2}^{3 D, i s o}\left(\omega, T_{c}, \Lambda\right)=\frac{e^{2}}{6 \hbar \xi_{0}} \sqrt{\frac{2}{\pi} \frac{k_{B} T_{c}}{\hbar \omega}} \mathcal{F}_{1,2}^{3 D, i s o}(W),
$$

where

$$
\mathcal{F}_{1,2}^{3 D, i s o}(W)=\frac{1}{\pi}\left(C \mp \frac{1}{2} D\right)
$$

As explained above, from the ratio of the experimental values $\sigma_{2} / \sigma_{1}$ at $T_{c}$ one can determine the parameter $W$, and the values of $\mathcal{F}_{1,2}$ can then be calculated from Eq. (22). The remaining unknown parameter $\xi_{0}$ can be obtained using Eq. (21) and either of the experimental values of $\sigma_{1}\left(T_{c}\right)$ or $\sigma_{2}\left(T_{c}\right)$.

It is also interesting to look at the plots of $\mathcal{F}_{1,2}(W)$ in Fig. 四 b). One can observe that $\mathcal{F}_{2}$ saturates to unity already at small values of $W \geq 2$. On the other hand, $\mathcal{F}_{1}$ is smaller than unity at any finite value of $W$, in conformity with the ratio $\left(\mathcal{F}_{2} / \mathcal{F}_{1}\right)=\left(\mathcal{S}_{2} / \mathcal{S}_{1}\right)$ at $T_{c}$. At this point it is useful to find the expected range of the values of $W$ encountered in the experiments. For the microwave frequencies in the range 1-100 GHz, with $\Lambda=0.5$, and $T_{c}=100 \mathrm{~K}$, one finds that $W$ is in the range $9-90$. According to Fig. $4(\mathrm{~b}), \mathcal{F}_{2} \approx 1$ in this range. This means that the cutoff makes no effect on $\mathcal{S}_{2}$, and only $\mathcal{S}_{1}$ is reduced, in conformity with the calculated curves shown in Fig. 3

\section{ANISOTROPY}

Most high- $T_{c}$ superconductors are anisotropic, some of them even having a high value of the anisotropy parameter $\gamma=\xi_{a b 0} / \xi_{c 0}$. Therefore, for practical purposes one needs adequate expressions for the $a c$ fluctuation conductivity. The real part of the fluctuation conductivity in the ab-plane is obtained using the Kubo formalism as in the isotropic case. One obtains

$$
\sigma_{1}^{3 D, \text { aniso }}=\left(\frac{2 e \hbar}{m_{a b} \alpha}\right)^{2} k_{B} T_{c} \sum_{\mathbf{k}} \frac{k_{x}^{2} \tau_{0} / 2}{\left(1+k_{a b}^{2} \xi_{a b}^{2}+k_{c}^{2} \xi_{c}^{2}\right)\left[\Omega^{2}+\left(1+k_{a b}^{2} \xi_{a b}^{2}+k_{c}^{2} \xi_{c}^{2}\right)^{2}\right]} .
$$

Taking $k_{x}^{2}=k_{a b}^{2} / 2$, and substituting the variables $q_{a b}=k_{a b} \xi_{a b}$ and $q_{c}=k_{c} \xi_{c}$, one can evaluate the sum in Eq. (23) by integration in the $q_{a b}$-plane, and along the $q_{c}$-axis

$$
\sigma_{1}^{3 D, \text { aniso }}\left(\omega, T, \Lambda_{a b}, \Lambda_{c}\right)=\frac{e^{2}}{8 \pi \hbar \xi_{0 c}}\left(\frac{\xi(T)}{\xi_{0}}\right)^{z-1} \int_{0}^{Q_{a b}} \int_{-Q_{c}}^{Q_{c}} \frac{q_{a b}^{3}}{\left(1+q_{a b}^{2}+q_{c}^{2}\right)\left[\Omega^{2}+\left(1+q_{a b}^{2}+q_{c}^{2}\right)^{2}\right]} d q_{a b} d q_{c},
$$

where we allowed a cutoff $Q_{a b}(T)=\sqrt{2} \Lambda_{a b} \xi_{a b}(T) / \xi_{0 a b}$ in the $q_{a b}$-plane, and a possibly different cutoff $Q_{c}(T)=$ $\Lambda_{c} \xi_{c}(T) / \xi_{0 c}$ along the $q_{c}$-axis. The dimensionless parameter $\Omega$ is the same as given by Eq. (6). We use the notation $\xi(T) / \xi_{0}$ for both $\xi_{a b}(T) / \xi_{0 a b}$ and $\xi_{c}(T) / \xi_{0 c}$.

We may briefly examine the $d c$ case $(\Omega=0)$. With no cutoff one obtains

$$
\sigma_{d c}^{3 D, \text { aniso }}\left(T, \Lambda_{a b, c} \rightarrow \infty\right)=\frac{e^{2}}{32 \hbar \xi_{0 c}}\left(\frac{\xi(T)}{\xi_{0}}\right)^{z-1}
$$

which reduces to the Aslamazov-Larkin result for $z=2$ (relaxational dynamics) and $\xi(T) / \xi_{0}$ taken in the Gaussian limit. Note that the fluctuation conductivity in the $a b$-plane depends on $\xi_{0 c}$. Finite cutoff parameters reduce the fluctuation conductivity when the temperature is increased above $T_{c}$

$$
\begin{aligned}
\sigma_{d c}^{B D, \text { aniso }}\left(T, \Lambda_{a b}, \Lambda_{c}\right)=\frac{e^{2}}{16 \pi \hbar \xi_{0 c}}\left(\frac{\xi(T)}{\xi_{0}}\right)^{z-1} & {\left[\arctan \left(Q_{c}\right)-\frac{Q_{a b}^{2} Q_{c}}{2\left(1+Q_{a b}^{2}\right)\left(1+Q_{a b}^{2}+Q_{c}^{2}\right)}\right.} \\
& \left.-\frac{2+3 Q_{a b}^{2}}{2\left(1+Q_{a b}^{2}\right)^{3 / 2}} \arctan \left(\frac{Q_{c}}{\sqrt{1+Q_{a b}^{2}}}\right)\right] .
\end{aligned}
$$

This expression has not been reported in the previous literature. The analysis of a $d c$ fluctuation conductivity is difficult because of the number of unknown parameters. 
The ac fluctuation conductivity can be obtained from the integral in Eq. (24) for the real part while the imaginary part is obtained by the procedure analogous to that of the isotropic case described in the preceding section

$$
\sigma_{2}^{3 D, \text { aniso }}\left(\omega, T, \Lambda_{a b}, \Lambda_{c}\right)=\frac{e^{2}}{8 \pi \hbar \xi_{0 c}}\left(\frac{\xi(T)}{\xi_{0}}\right)^{z-1} \int_{0}^{Q_{a b}} \int_{-Q_{c}}^{Q_{c}} \frac{\Omega q_{a b}^{3}}{\left(1+q_{a b}^{2}+q_{c}^{2}\right)^{2}\left[\Omega^{2}+\left(1+q_{a b}^{2}+q_{c}^{2}\right)^{2}\right]} d q_{a b} d q_{c}
$$

The full expression can again be written in the form

$$
\tilde{\sigma}^{3 D, \text { aniso }}\left(\omega, T, \Lambda_{a b}, \Lambda_{c}\right)=\frac{e^{2}}{32 \hbar \xi_{0 c}}\left(\frac{\xi(T)}{\xi_{0}}\right)^{z-1}\left[\mathcal{S}_{1}^{3 D, \text { aniso }}\left(\omega, T, \Lambda_{a b}, \Lambda_{c}\right)+i \mathcal{S}_{2}^{3 D, \text { aniso }}\left(\omega, T, \Lambda_{a b}, \Lambda_{c}\right)\right]
$$

The $\mathcal{S}$-functions for the $3 D$ anisotropic case are found to be

$$
\begin{aligned}
& \mathcal{S}_{1}^{3 D, \text { aniso }}\left(\omega, T, \Lambda_{a b}, \Lambda_{c}\right)=\frac{1}{3 \pi \Omega^{2}}\left[2 Q_{c}\left(3+Q_{c}^{2}\right) L_{1}+P_{-}\left(P_{+}^{2}+2\right) L_{2}-T_{-}\left(T_{+}^{2}+2-Q_{a b}^{2}\right) L_{3}+2 P_{+}\left(P_{-}^{2}-2\right) A_{1}\right. \\
& \left.-2 T_{+}\left(T_{-}^{2}-2+Q_{a b}^{2}\right) A_{2}-8 \sqrt{1+Q_{a b}^{2}}\left(2-Q_{a b}^{2}\right) A_{3}+16 A_{4}+12 \Omega Q_{c} A_{5}\right] \text {, } \\
& \mathcal{S}_{2}^{3 D, \text { aniso }}\left(\omega, T, \Lambda_{a b}, \Lambda_{c}\right)=\frac{1}{3 \pi \Omega^{2}}\left[4 Q_{c}\left(3+Q_{c}^{2}\right) A_{5}+2 P_{-}\left(P_{+}^{2}+2\right) A_{1}-2 T_{-}\left(T_{+}^{2}+2-Q_{a b}^{2}\right) A_{2}-P_{+}\left(P_{-}^{2}-2\right) L_{2}\right. \\
& \left.+T_{+}\left(T_{-}^{2}-2+Q_{a b}^{2}\right) L_{3}+12 \Omega \frac{2+Q_{a b}^{2}}{\sqrt{1+Q_{a b}^{2}}} A_{3}-24 \Omega A_{4}-6 \Omega Q_{c} L_{1}\right],
\end{aligned}
$$

where we used the shorthand notations for $P_{ \pm}$as in Eq. (14), and the following

$$
\begin{gathered}
T_{ \pm}=\sqrt{2} \sqrt{\sqrt{\left(1+Q_{a b}^{2}\right)^{2}+\Omega^{2}} \pm\left(1+Q_{a b}^{2}\right)} \\
L_{1}=\ln \left(\frac{\left(1+Q_{c}^{2}\right)^{2}\left[\Omega^{2}+\left(1+Q_{a b}^{2}+Q_{c}^{2}\right)^{2}\right]}{\left(1+Q_{a b}^{2}+Q_{c}^{2}\right)^{2}\left[\Omega^{2}+\left(1+Q_{c}^{2}\right)^{2}\right]}\right) \\
L_{2}=\ln \left(\frac{2+Q_{c}^{2}+\left(Q_{c}-P_{-}\right)^{2}}{\left.2+Q_{c}^{2}+\left(Q_{c}+P_{-}\right)^{2}\right)}\right) \\
L_{3}=\ln \left(\frac{2\left(1+Q_{a b}^{2}\right)+Q_{c}^{2}+\left(Q_{c}-T_{-}\right)^{2}}{2\left(1+Q_{a b}^{2}\right)+Q_{c}^{2}+\left(Q_{c}+T_{-}\right)^{2}}\right) \\
A_{1}=\arctan \left(\frac{2 Q_{c}+P_{-}}{P_{+}}\right)+\arctan \left(\frac{2 Q_{c}-P_{-}}{P_{+}}\right) \\
A_{3}=\arctan \left(\frac{2 Q_{c}+T_{-}}{T_{+}}\right)+\arctan \left(\frac{2 Q_{c}-T_{-}}{T_{+}}\right) \\
\left.A_{4}\right)
\end{gathered}
$$


The effects of cutoff are similar as those described at length in the preceding section for the simpler case of $3 D$ isotropic superconductors. In this section we discuss only the modifications in the limit $T \rightarrow T_{c}$ where the relevant parameters can be determined. The $\mathcal{S}$-functions can be expanded in the limit of $T_{c}(\Omega \rightarrow \infty)$, and the leading terms are

$$
\begin{aligned}
& \mathcal{S}_{1}^{3 D, \text { aniso }}\left(W_{a b}, W_{c}, \Omega \rightarrow \infty\right) \approx \frac{4 \sqrt{2}}{3 \pi} \quad\left[C_{1}-U_{+}\left(\sqrt{1+W_{a b}^{4}}-\frac{1}{2} W_{a b}^{2}\right) C_{2}+\frac{3}{\sqrt{2}} W_{c} C_{3}+\sqrt{2} W_{a b}^{3} C_{4}\right. \\
& \left.-\frac{1}{2} D_{1}+\frac{1}{2} U_{-}\left(\sqrt{1+W_{a b}^{4}}+\frac{1}{2} W_{a b}^{2}\right) D_{2}+\frac{1}{2 \sqrt{2}} W_{c}^{3} D_{3}\right] \frac{1}{\sqrt{\Omega}}, \\
& \mathcal{S}_{2}^{3 D, a n i s o}\left(W_{a b}, W_{c}, \Omega \rightarrow \infty\right) \approx \frac{4 \sqrt{2}}{3 \pi}\left[C_{1}-U_{-}\left(\sqrt{1+W_{a b}^{4}}+\frac{1}{2} W_{a b}^{2}\right) C_{2}+\frac{1}{\sqrt{2}} W_{c}^{3} C_{3}+\frac{3}{\sqrt{2}} W_{a b} C_{4}\right. \\
& \left.+\frac{1}{2} D_{1}-\frac{1}{2} U_{+}\left(\sqrt{1+W_{a b}^{4}}-\frac{1}{2} W_{a b}^{2}\right) D_{2}-\frac{3}{2 \sqrt{2}} W_{c} D_{3}\right] \frac{1}{\sqrt{\Omega}},
\end{aligned}
$$

where we used the following shorthand notations

$$
\begin{gathered}
U_{ \pm}=\sqrt{\sqrt{1+W_{a b}^{4}} \pm W_{a b}^{2}}, \\
C_{1}=\arctan \left(1+\sqrt{2} W_{c}\right)-\arctan \left(1-\sqrt{2} W_{c}\right), \\
C_{2}=\arctan \left(\frac{U_{-}+\sqrt{2} W_{c}}{U_{+}}\right)-\arctan \left(\frac{U_{-}-\sqrt{2} W_{c}}{U_{+}}\right), \\
C_{3}=\arctan \left(W_{a b}^{2}+W_{c}^{2}\right)-\arctan \left(W_{c}^{2}\right), \\
C_{4}=\arctan \left(\frac{W_{c}}{W_{a b}}\right), \\
D_{1}=\ln \left(\frac{1+\sqrt{2} W_{c}+W_{c}^{2}}{1-\sqrt{2} W_{c}+W_{c}^{2}}\right), \\
D_{3}=\ln \left(\frac{W_{c}^{4}\left[1+\left(W_{a b}^{2}+W_{c}^{2}\right)^{2}\right]}{\left(W_{a b}^{2}+W_{c}^{2}\right)^{2}\left(1+W_{c}^{4}\right)}\right) . \\
\left.\sqrt{1+W_{a b}^{4}+\sqrt{2} W_{c} U_{-}+W_{c}^{2}}\right),
\end{gathered}
$$

The cutoff parameters appear in

$$
\begin{gathered}
W_{a b}=\sqrt{2} \Lambda_{a b} \sqrt{\frac{16}{\pi} \frac{k_{B} T_{c}}{\hbar \omega}}, \\
W_{c}=\Lambda_{c} \sqrt{\frac{16}{\pi} \frac{k_{B} T_{c}}{\hbar \omega}} .
\end{gathered}
$$


We note that in the anisotropic case, the $\mathcal{S}$-functions behave also as $1 / \sqrt{\Omega}$ when $T \rightarrow T_{c}$. As already discussed in the previous section, this implies that finite nonzero $\sigma_{1}\left(T_{c}\right)$ and $\sigma_{2}\left(T_{c}\right)$ can be obtained only for $z=2$ (relaxational model). Since the available experimental data in anisotropic high- $T_{c}$ superconductors ${ }^{6.7 .8}$ show finite nonzero $\sigma_{1}\left(T_{c}\right)$ and $\sigma_{2}\left(T_{c}\right)$, we can adopt $z=2$ in the remainder of this section.

In analogy to the $3 D$ isotropic case described in the preceding section, one may define the functions

$$
\mathcal{F}_{1,2}^{3 D, \text { aniso }}\left(W_{a b}, W_{c}\right)=\frac{3 \sqrt{\Omega}}{4 \sqrt{2}} \mathcal{S}_{1,2}^{3 D, \text { aniso }}\left(W_{a b}, W_{c}, \Omega \rightarrow \infty\right)
$$

so that the conductivities at $T_{c}$ are given by

$$
\sigma_{1,2}^{3 D, \text { aniso }}\left(\omega, T_{c}, \Lambda_{a b}, \Lambda_{c}\right)=\frac{e^{2}}{6 \hbar \xi_{0 c}} \sqrt{\frac{2}{\pi} \frac{k_{B} T_{c}}{\hbar \omega}} \mathcal{F}_{1,2}^{3 D, \text { aniso }}\left(W_{a b}, W_{c}\right) .
$$

The ratio of experimental values $\sigma_{2} / \sigma_{1}$ at $T_{c}$ does not define uniquely the cutoff parameters $\Lambda_{a b}$ and $\Lambda_{c}$. It puts, however, a constraint on their choice. Fig. [5(a) shows the plot of $\mathcal{F}_{2} / \mathcal{F}_{1}$ given by Eq. (52) as a function of two variables, $W_{a b}$ and $W_{c}$. It is evident that a fixed value of $\mathcal{F}_{2} / \mathcal{F}_{1}$ defines a simple curve of the possible choices of

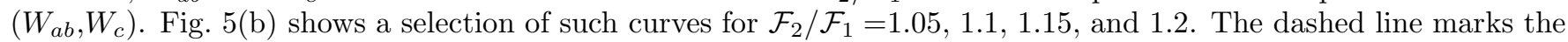
condition $\Lambda_{a b}=\Lambda_{c}\left(W_{a b}=\sqrt{2} W_{c}\right)$. Experimentally, one has to probe the possible choices for $\left(W_{a b}, W_{c}\right)$ and look at the fits of the theoretical curves to the experimental data at $T>T_{c}$. The parameter $\xi_{0 c}$ in Eq. (28) can be obtained once the choice for $\left(W_{a b}, W_{c}\right)$ is made.

Note that in practical applications of the above theory one needs measurements where the microwave current flows only in the ab-plane. Particularly suitable for this purpose are the measurements in which the superconducting sample is placed in the antinode of the microwave electric field $E_{\omega}$ in the cavity ${ }^{34.35}$

\section{IV. $2 D$ FLUCTUATIONS}

Superconducting transition does not occur in a strictly $2 D$ system. However, if the sample is a very thin film so that its thickness is much smaller than the correlation length, the fluctuations will be restricted within the film thickness

$d$ in one direction, and develop freely only in the plane of the film. Using the formalism described in the preceding sections, we find that the fluctuation conductivity is given by

$$
\begin{gathered}
\sigma_{1}^{2 D}(\omega, T, \Lambda)=\frac{e^{2}}{4 \hbar d}\left(\frac{\xi(T)}{\xi_{0}}\right)^{2} \sum_{q_{n}} \int_{0}^{Q} \frac{q^{3}}{\left(1+q^{2}+q_{n}^{2}\right)\left[\Omega^{2}+\left(1+q^{2}+q_{n}^{2}\right)^{2}\right]} d q \\
\sigma_{2}^{2 D}(\omega, T, \Lambda)=\frac{e^{2}}{4 \hbar d}\left(\frac{\xi(T)}{\xi_{0}}\right)^{2} \sum_{q_{n}} \int_{0}^{Q} \frac{\Omega q^{3}}{\left(1+q^{2}+q_{n}^{2}\right)^{2}\left[\Omega^{2}+\left(1+q^{2}+q_{n}^{2}\right)^{2}\right]} d q \\
\tilde{\sigma}^{2 D}(\omega, T, \Lambda)=\frac{e^{2}}{16 \hbar d}\left(\frac{\xi(T)}{\xi_{0}}\right)^{2} \sum_{q_{n}}\left[\mathcal{S}_{1}^{2 D}\left(\Omega, Q, q_{n}\right)+i \mathcal{S}_{2}^{2 D}\left(\Omega, Q, q_{n}\right)\right]
\end{gathered}
$$

where

$$
q_{n}=n \pi\left(\frac{\xi_{0}}{d}\right)\left(\frac{\xi(T)}{\xi_{0}}\right)
$$

and $Q=\sqrt{2} \Lambda\left(\xi(T) / \xi_{0}\right)$. The prefactor is the Aslamazov-Larkin result for the $2 D$ case with no cutoff, and the Gaussian form $1 / \epsilon$ replaced by the more general expression $\left(\xi(T) / \xi_{0}\right)^{2}$.

The $\mathcal{S}$-functions are given by

$$
\begin{aligned}
\mathcal{S}_{1}^{2 D}\left(\Omega, Q, q_{n}\right)=\frac{1}{\Omega} & {\left[2 \arctan \left(\frac{1+Q^{2}+q_{n}^{2}}{\Omega}\right)-2 \arctan \left(\frac{1+q_{n}^{2}}{\Omega}\right)\right.} \\
+ & \left.\left(\frac{1+q_{n}^{2}}{\Omega}\right) \ln \left(\frac{\left(1+q_{n}^{2}\right)^{2}\left[\Omega^{2}+\left(1+Q^{2}+q_{n}^{2}\right)^{2}\right]}{\left(1+Q^{2}+q_{n}^{2}\right)^{2}\left[\Omega^{2}+\left(1+q_{n}^{2}\right)^{2}\right]}\right)\right],
\end{aligned}
$$




$$
\begin{aligned}
\mathcal{S}_{2}^{2 D}\left(\Omega, Q, q_{n}\right)=\frac{1}{\Omega} & \quad\left[\frac{2\left(1+q_{n}^{2}\right)}{\Omega}\left[\arctan \left(\frac{1+Q^{2}+q_{n}^{2}}{\Omega}\right)-\arctan \left(\frac{1+q_{n}^{2}}{\Omega}\right)\right]\right. \\
& \left.-\ln \left(\frac{\left(1+q_{n}^{2}\right)^{2}\left[\Omega^{2}+\left(1+Q^{2}+q_{n}^{2}\right)^{2}\right]}{\left(1+Q^{2}+q_{n}^{2}\right)^{2}\left[\Omega^{2}+\left(1+q_{n}^{2}\right)^{2}\right]}\right)-2 \frac{Q^{2}}{1+Q^{2}+q_{n}^{2}}\right] .
\end{aligned}
$$

The summation over $q_{n}$ in Eq. (56) has to carried out until the factor $n \pi\left(\xi_{0} / d\right)$ reaches some cutoff value $\Lambda$, which is of the order of unity. If the film thickness is large $\left(d \gg \xi_{0}\right)$, one has to sum up to a high $n$-value. In such cases, the summation is well approximated by an integration, and one retrieves the $3 D$ case of the preceding section. The $2 D$ character is better displayed when the film thickness is comparable to $\xi_{0}$. Then, only a few terms have to be taken into account. In the extreme case of $d<\xi_{0}$, only the $n=0$ term is found below the cutoff limit.

The zero frequency limit $(\Omega \rightarrow 0)$ yields

$$
\sigma_{d c}^{2 D}(T, \Lambda)=\frac{e^{2}}{16 \hbar d}\left(\frac{\xi(T)}{\xi_{0}}\right)^{2} \sum_{q_{n}} \frac{Q^{4}}{\left(1+Q^{2}+q_{n}^{2}\right)^{2}\left(1+q_{n}^{2}\right)} .
$$

The $n=0$ term yields the previous result of Hopfengärtner et al $\stackrel{4}{\underline{\underline{n}}}$ and Gauzzi et al ${ }^{\underline{5}}$

In the limit of $T_{c}(\Omega \rightarrow \infty)$ one obtains

$$
\begin{gathered}
\sigma_{1}^{2 D}\left(T_{c}\right)=\frac{e^{2}}{\pi \hbar d} \frac{k_{B} T_{c}}{\hbar \omega} \sum_{n}\left[2 \arctan \left(W^{2}+W_{n}^{2}\right)-2 \arctan \left(W_{n}^{2}\right)+W_{n}^{2} \ln \frac{W_{n}^{4}\left[1+\left(W^{2}+W_{n}^{2}\right)^{2}\right]}{\left(1+W_{n}^{4}\right)\left(W^{2}+W_{n}^{2}\right)^{2}}\right], \\
\sigma_{2}^{2 D}\left(T_{c}\right)=\frac{e^{2}}{\pi \hbar d} \frac{k_{B} T_{c}}{\hbar \omega} \sum_{n}\left[2 W_{n}^{2} \arctan \left(W^{2}+W_{n}^{2}\right)-2 W_{n}^{2} \arctan \left(W_{n}^{2}\right)-\ln \frac{W_{n}^{4}\left[1+\left(W^{2}+W_{n}^{2}\right)^{2}\right]}{\left(1+W_{n}^{4}\right)\left(W^{2}+W_{n}^{2}\right)^{2}}\right],
\end{gathered}
$$

where we used the notation

$$
\begin{gathered}
W=\sqrt{2} \Lambda \sqrt{\frac{16}{\pi} \frac{k_{B} T_{c}}{\hbar \omega}}, \\
W_{n}=n \pi\left(\frac{\xi_{0}}{d}\right) \sqrt{\frac{16}{\pi} \frac{k_{B} T_{c}}{\hbar \omega}} .
\end{gathered}
$$

One may observe that for $n=0$ the real part of the conductivity is finite, but the imaginary part diverges. It is due to the logarithmic term in Eq. (62). This is an unphysical result. It may indicate that the $n=0$ term is not physically acceptable, or that the $2 D$ model should not be applied exactly at $T_{c}$.

\section{COMPARISON WITH EXPERIMENT}

The relevance of the theoretical expressions derived in the preceding sections can be demonstrated by comparison of the calculated and experimentally measured $a c$ fluctuation conductivity. As an example we present here an analysis of the data in $\mathrm{Bi}_{2} \mathrm{Sr}_{2} \mathrm{Ca}_{2} \mathrm{Cu}_{3} \mathrm{O}_{10-\delta}$ thin film. The experimental results of the complex conductivity measured at $9.5 \mathrm{GHz}$ are shown in Fig. 6(a). The main features are the same as reported previously in single crystals of high$T_{c}$ superconductors 6.18 We have to note that in our measurement the thin film was positioned in the center of an elliptical microwave cavity resonating in $\mathrm{TE}_{111}$ mode, and oriented in such a way that the electric field $E_{\omega}$ was in the $a b$-plane. Thus the in-plane conductivity was measured and the application of the theoretical expressions of the preceding sections is appropriate. Other experimental details have been reported previously 35.36 .37 In this section we are interested in the fluctuation conductivity near $T_{c}$ which is shown on an enlarged scale in Fig. 6(b). The real part of the conductivity has a maximum when the coherence length diverges. Since the critical temperature of a phase transition is characterized by the divergence of the coherence length, we use the maximum of $\sigma_{1}$ in Fig. 6(b) to determine $T_{c}=84.04 \mathrm{~K}$. One can also observe in Fig. 6 b) that the imaginary part of the conductivity crosses the real part at a temperature slightly above $T_{c}$. This is a direct experimental evidence of the short wavelength cutoff as discussed in Section III The experimental values of $\sigma_{1}$ and $\sigma_{2}$ at $T_{c}$ can be used in the evaluation of the parameters which enter the theoretical expressions of the preceding sections. 
Fig. 17(a) shows the experimental data above $T_{c}$ plotted against the reduced temperature $\epsilon=\ln \left(T / T_{c}\right)$. We can analyze this data first by the theoretical expressions which have no cutoff on the fluctuation wavevector. If one sets $\Lambda_{a b}=\Lambda_{c}=\infty$ for the $3 D$ anisotropic case, it is straightforward to evaluate $\xi_{0 c}$ using Eq. (53) and the experimental value of $\sigma_{2}$ at $T_{c}$. We have obtained $\xi_{0 c}=0.016 \mathrm{~nm}$ in $B i_{2} S r_{2} C a_{2} C u_{3} O_{10-\delta}$ thin film. Once this parameter is determined, the fluctuation conductivity at all temperatures above $T_{c}$ follows from Eq. (28). The real and imaginary parts of the $a c$ fluctuation conductivity have to be mutually consistent. This can be exploited in the data analysis. We insert the experimental values of $\sigma_{2}$ into the imaginary part of Eq. (28), and solve numerically for $\xi(T) / \xi_{0}$. Then we exploit these same values of the reduced correlation length in the real part of Eq. (28). The calculated $\sigma_{1}$ is shown by the dotted line in Fig. 7(a). The calculated line lies far from the experimental points. Note in particular that the calculated $\sigma_{1}$ meets $\sigma_{2}$ at $T_{c}$ when no cutoff is included (cf. Section II). Besides, the shape of the calculated $\sigma_{1}$ differs from that of the experimental one. We may conclude that with no cutoff on the fluctuation wavevector the theoretical expression does not describe properly the experimental fluctuation conductivity.

A finite cutoff on the fluctuation wavevector improves greatly the agreement of the theory and experiment. From Fig. 6] (b) we can evaluate the ratio $\sigma_{2}\left(T_{c}\right) / \sigma_{1}\left(T_{c}\right)=1.28$ at $T_{c}$. This value yields a constraint on the choices of $\Lambda_{a b}$ and $\Lambda_{c}$ as described in Section III and Fig. 5 The actual choices are presented in the inset of Fig. 7 (b). For a given choice $\left(\Lambda_{a b}, \Lambda_{c}\right)$ from this constraining line, one has to determine first $\xi_{0 c}$ using Eq. (53) and the experimental value of $\sigma_{2}$ at $T_{c}$. Then, the temperature dependence of the reduced correlation length $\xi(T) / \xi_{0}$ is evaluated numerically from the imaginary part of Eq. (28) with the selected pair $\left(\Lambda_{a b}, \Lambda_{c}\right)$ and the experimental values of $\sigma_{2}$. The obtained values of $\xi(T) / \xi_{0}$ are finally used to calculate $\sigma_{1}$ from the real part of Eq. (28). The results vary with the possible choices of the pairs $\left(\Lambda_{a b}, \Lambda_{c}\right)$ from inset of Fig. [ (b). The best fit of the calculated $\sigma_{1}$ to the experimental points is shown by the solid line in Fig. 7f(a). It is obtained with the choice $\left(\Lambda_{a b}=0.71, \Lambda_{c}=0.05\right)$. It is physically reasonable. With $\Lambda_{a b}$ being of the order of unity, the minimum wavelength of the superconducting fluctuations in the $a b$-plane is given by $2 \pi \xi_{0 a b}$, which is much larger than the atomic size and could be accepted as a mesoscopic quantity. In contrast, the value of $2 \pi \xi_{0 c}$ is below the atomic size and, hence, could not be physically accepted for the lower limit of the fluctuation wavelength along the c-axis. Therefore one needs a value of $\Lambda_{c}<<1$ so that the minimum wavelength of the superconducting fluctuations $2 \pi \xi_{0 c} / \Lambda_{c}$ along the c-axis becomes also an acceptable mesoscopic quantity.

We have tested also a number of other choices of the cutoff parameters. By shifting the choice of the parameters along the constraining curve in the inset of Fig. 7b, one degrades the fit of the calculated $\sigma_{1}$ to the experimental points. The dashed line in Fig. 7( $(\mathrm{a})$ is the calculated $\sigma_{1}$ using the choice of equal cutoff parameters $\left(\Lambda_{a b}=\Lambda_{c}=0.09\right.$ ) permitted by the constraint in the inset of Fig. 7(b). The fit to the experimental points is seen to be much worse than that of the full line. For the sake of completeness, we show also the result for a choice of the cutoff parameters on the other branch of the constraining curve in the inset of Fig. 7 (b). If one takes $\left(\Lambda_{a b}=0.08, \Lambda_{c}=0.71\right)$ the calculated $\sigma_{1}$ is as shown by the dashed-dotted line in Fig. 7(a). The fit is unsatisfactory. Moreover, this choice has to be refuted on the grounds of physically unacceptable minimum wavelength of the fluctuations along the c-axis. Also shown in Fig. 7(a) by the short-dotted line is the result obtained by the isotropic 3D expression in Eq. (11). In this case the parameters $\xi_{0}=0.016 \mathrm{~nm}$ and $\Lambda=0.08$ are obtained straightforwardly from Eq. (21) and the experimental values of $\sigma_{1}$ and $\sigma_{2}$ at $T_{c}$. The fit in Fig. 7(a) is obviously not good. It is also seen that the expressions for the anisotropic $3 D$ case always yield curves which are different from that of the isotropic $3 D$ case. The complexity of the anisotropic 3D expressions elaborated in Section III is not futile. Indeed, we find that these expressions must be used when analyzing an anisotropic superconductor. Fig. 7(b) shows an enlarged view of the high temperature part where the same curves as in Fig. 7(a) are better distinguished.

We have analyzed also the 2D expressions of Section IV Fig. 8) (a) shows again the same experimental data as in Fig. [7(a), but fitted with $n=0$ term of the $2 D$ expansion in Eq. (56). The parameter $d$ has been chosen so as to optimize the fit to the experimental values of $\sigma_{1}$. The resulting curve in Fig. 8 (a) was obtained with $d=1.2$ nm. The $2 D$ results are not so sensitive to the fluctuation wavevector cutoff as those of the $3 D$ case. The curves obtained with no cutoff $(\Lambda=\infty)$ and with $\Lambda=0.7$ are practically indistinguishable in Fig. 8 (a). One may conclude that closer to $T_{c}$ the BSCCO superconductor clearly does not behave as a $2 D$ system. However, at higher temperatures both, $2 D$ and $3 D$ expressions yield almost equally good fits to the experimental values, as seen in Fig. 8 (b). Thus the dimensionality of the fluctuations at higher temperatures cannot be resolved from the ac fluctuation conductivity.

Finally, we remark that the above analysis could explain very well the experimental ac fluctuation conductivity above $T_{c}$ in the BSCCO thin film using the Aslamazov-Larkin type expressions with wavevector cutoff as deduced in the preceding sections of this paper. The other contributions such as Maki-Thomson and one electron density of states, mentioned in Section II are not necessary over most of the temperature range covered in the experiment. This is in accord with the recent microscopic calculation ${ }^{38}$ proving that these contributions may cancel in the ultraclean case of nonlocal electrodynamics. However, they may play a role at high enough temperatures where the above calculated curves depart from the experimental data. Their analysis is beyond the scope of the present paper. 


\section{CONCLUSIONS}

We have presented full analytical expressions for the ac fluctuation conductivity in $3 D$ isotropic, 3D anisotropic, and $2 D$ superconductors. The effects of the short wavelength cutoff in the fluctuation spectrum on the $d c$ and $a c$ conductivities were discussed in detail. The short wavelength cutoff brings about a breakdown of the scaling property in frequency and temperature. It also has a small, but experimentally very important effect on $\sigma_{1}$. Due to a finite cutoff parameter $\Lambda$, the value of $\sigma_{1}$ at $T_{c}$ is lower than that of $\sigma_{2}$. In the simpler $3 D$ isotropic case, this observation can be used to determine $\Lambda$ directly from the experimental data at $T_{c}$. In the $3 D$ anisotropic case, one obtains a constraint on the choices of $\left(\Lambda_{a b}, \Lambda_{c}\right)$.

The useful feature of ac fluctuation conductivity measurements is that $T_{c}$ can be determined directly from the experimental data. Moreover, the expressions derived in this paper enable the determination of $\xi_{0}$ ( $3 D$ isotropic), or $\xi_{c 0}$ (3D anisotropic) from the experimental values $\sigma_{1,2}\left(T_{c}\right)$. Thus, we establish that the analysis of $a c$ fluctuation conductivity requires no free fit parameters in the $3 D$ isotropic case, and only one $\left(\Lambda_{a b}, \Lambda_{c}\right)$ in the $3 D$ anisotropic case.

We have shown that the anisotropic $3 D$ expressions with an appropriate cutoff of the fluctuation wavevector can account for the experimental fluctuation conductivity in a BSCCO thin film within a large temperature range above $T_{c}$. The $2 D$ expression is less sensitive to the cutoff and was found to match the experimental data only at higher temperatures.

\section{Acknowledgments}

D.-N. Peligrad and M. Mehring acknowledge support by the Deutsche Forschungsgemeinschaft (DFG) project Nr. Me362/14-2. A. Dulčić acknowledges support from the Croatian Ministry of Science, and DLR Stiftung (project Nr. KRO-004-98).

* present address: Philips Research Laboratories, Weisshausstrasse 2, D-52066, Aachen, Germany

$¥$ lectronic address: D.-N. Peligrad (dragos.peligrad@philips.com); M. Mehring (m.mehring@physik.uni-stuttgart.de); A. Dulčić (dulcic@faust.irb.hr)

1 C. J. Lobb, Phys. Rev. B36, 3930 (1987).

${ }^{2}$ W. J. Skocpol and M. Tinkham, Rep. Prog. Phys. 38, 1049 (1975).

3 M. Ausloos and Ch. Laurent, Phys. Rev. B 37, 611 (1988).

${ }^{4}$ R. Hopfengärtner, B. Hensel, and G. Saemann-Ischenko, Phys. Rev. B 44, 741 (1991).

5 A. Gauzzi and D. Pavuna, Phys. Rev. B 51, 15420 (1995).

6 S. M. Anlage, J. Mao, J. C. Booth, D. H. Wu, and J. L. Peng, Phys. Rev. B 53, 2792 (1996).

7 J. C. Booth, D. H. Wu, S. B. Qadri, E. F. Skelton, M. S. Osofsky, A. Piqué, and S. M. Anlage, Phys. Rev. Lett 77, 4438 (1996).

8 J. Kötzler and M. Kaufmann, Phys. Rev. B 56, 13734 (1997).

9 M. R. Cimberle, C. Ferdeghini, E. Giannini, D. Marré, M.Putti, A. Siri, F. Federici, and A. Varlamov, Phys. Rev. B 55, R14745 (1997).

10 R. M. Costa, P. Pureur, L. Ghivelder, J. A. Campá, and I. Rasines, Phys. Rev. B 56, 10836 (1997).

11 S. H. Han, Yu. Eltsev, and Ö. Rapp, Phys. Rev. B 57, 7510 (1998).

12 S. H. Han, Yu. Eltsev, and Ö. Rapp, Phys. Rev. B 61, 11776 (2000).

13 E. Silva, S. Sarti, R. Fastampa, and M. Giura, Phys. Rev. B 64, 144508 (2001).

14 R. M. Costa, P. Pureur, M. Gusmão, S. Senoussi, and K. Behnia, Phys. Rev. B 64, 214513 (2001).

15 L. Reggiani, R. Vaglio, and A. A. Varlamov, Phys. Rev. B 44, 9541 (1991).

16 G. Nakielski, D. Görlitz, Chr. Stodte, M. Welters, A. Krämer, and J.Kötzler, Phys. Rev. B 55, 6077 (1997).

17 D. Neri, E. Silva, S. Sarti, R.Marcon, M. Giura, R. Fastampa, and N. Sparvieri, Phys. Rev. B 58, 14581 (1998).

18 J. R. Waldram, D. M. Broun, D. C. Morgan, R. Ormeno, and A. Porch, Phys. Rev. B 59, 1528 (1999).

19 O. Klein, E. J. Nicol, K. Holczer, and G. Grüner, Phys. Rev. B 50, 6307 (1994).

${ }^{20}$ H. Schmidt, Z. Phys. 216, 336 (1968).

21 D. S. Fisher, M. P. A. Fisher, and D. A. Huse, Phys. Rev. B 43, 130 (1991).

22 A. T. Dorsey, Phys. Rev. B 43, 7575 (1991).

23 R. A. Wickham and A. T. Dorsey, Phys. Rev. B 61, 6945 (2000).

24 B. R. Patton, V. Ambegaokar, and J. W. Wilkins, Solid State Commun. 7, 1287 (1969).

25 J. P. Gollub, M. R. Beasley, R. Callarotti, and M. Tinkham, Phys. Rev. B 7, 3039 (1973).

${ }^{26}$ W. L. Johnson, C. C. Tsuei, and P. Chaudhari, Phys. Rev. B 17, 2884 (1978). 
27 M. Tinkham, Introduction to Superconductivity, 2nd ed. (McGraw-Hill, New York, 1995).

28 D. Neri, R. Marcon, E. Silva, R. Fastampa, L. Iacobucci, and S. Sarti, Int. J. Mod. Phys. B 13, 1097 (1999).

29 D. Neri, R. Fastampa, M. Giura, R. Marcon, S. Sarti, and E. Silva, J. Low Temp. Phys. 117, 1099 (1999).

30 L. G. Aslamazov and A. A. Varlamov, J. Low Temp. Phys. 38, 223 (1980).

31 F. Federici and A. A. Varlamov, Phys. Rev. B 55, 6070 (1997).

32 A. I. Larkin and A. A. Varlamov, in "Handbook on Superconductivity: Conventional and Unconventional Superconductors", edited by K.-H.Bennemann and J.B. Ketterson, Springer, Berlin 2002.

33 A. A. Varlamov, G. Balestrino, E. Milani, and D. V. Livanov, Adv. Phys. 48, 655 (1999).

34 D.-N. Peligrad, B. Nebendahl, C. Kessler, M. Mehring, A. Dulčić, M. Požek, and D. Paar, Phys. Rev. B 58, 11652 (1998).

35 D.-N. Peligrad, B. Nebendahl, M. Mehring, A. Dulčić, M. Požek, and D. Paar, Phys. Rev. B 64, 224504 (2001).

36 B. Nebendahl et al., Rev. Sci. Instr. 72, 1876 (2001).

37 D.-N. Peligrad, B. Nebendahl, M. Mehring, A. Dulčić, submitted to Phys. Rev. Lett., cond. mat./0209176.

38 D. V. Livanov, G. Savona, and A. A. Varlamov, Phys. Rev. B 62, 8675 (2000). 

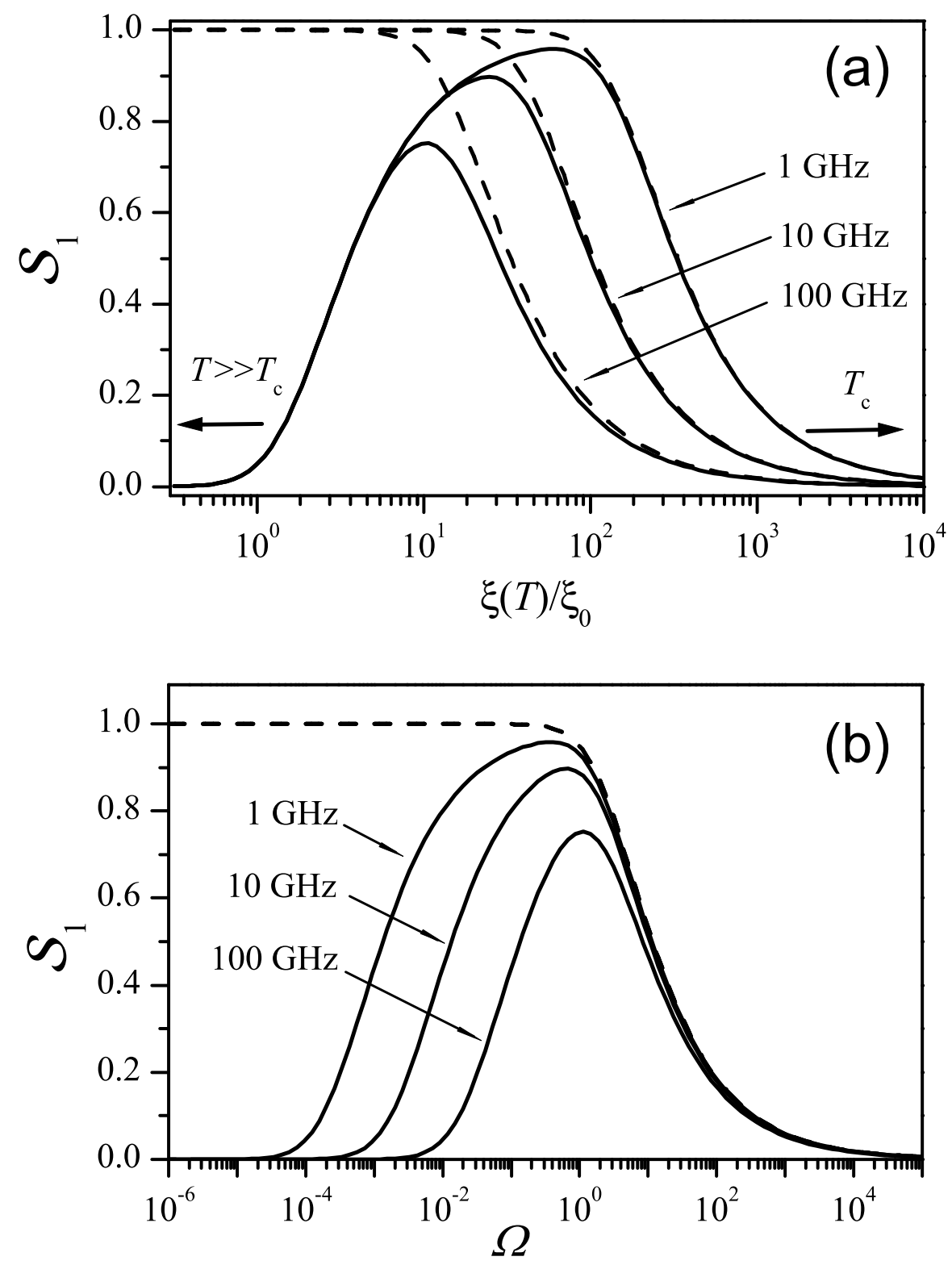

FIG. 1: $\mathcal{S}_{1}$-curves for the $3 D$ isotropic case calculated from Eq. (12) for a finite cutoff parameter $\Lambda=0.5$ (full lines), and for $\Lambda=\infty$ (dashed lines). The variable is $\xi(T) / \xi_{0}$ in (a), and $\Omega$ in (b). The curves are labelled by the frequency $\omega / 2 \pi$ used in the calculations. 

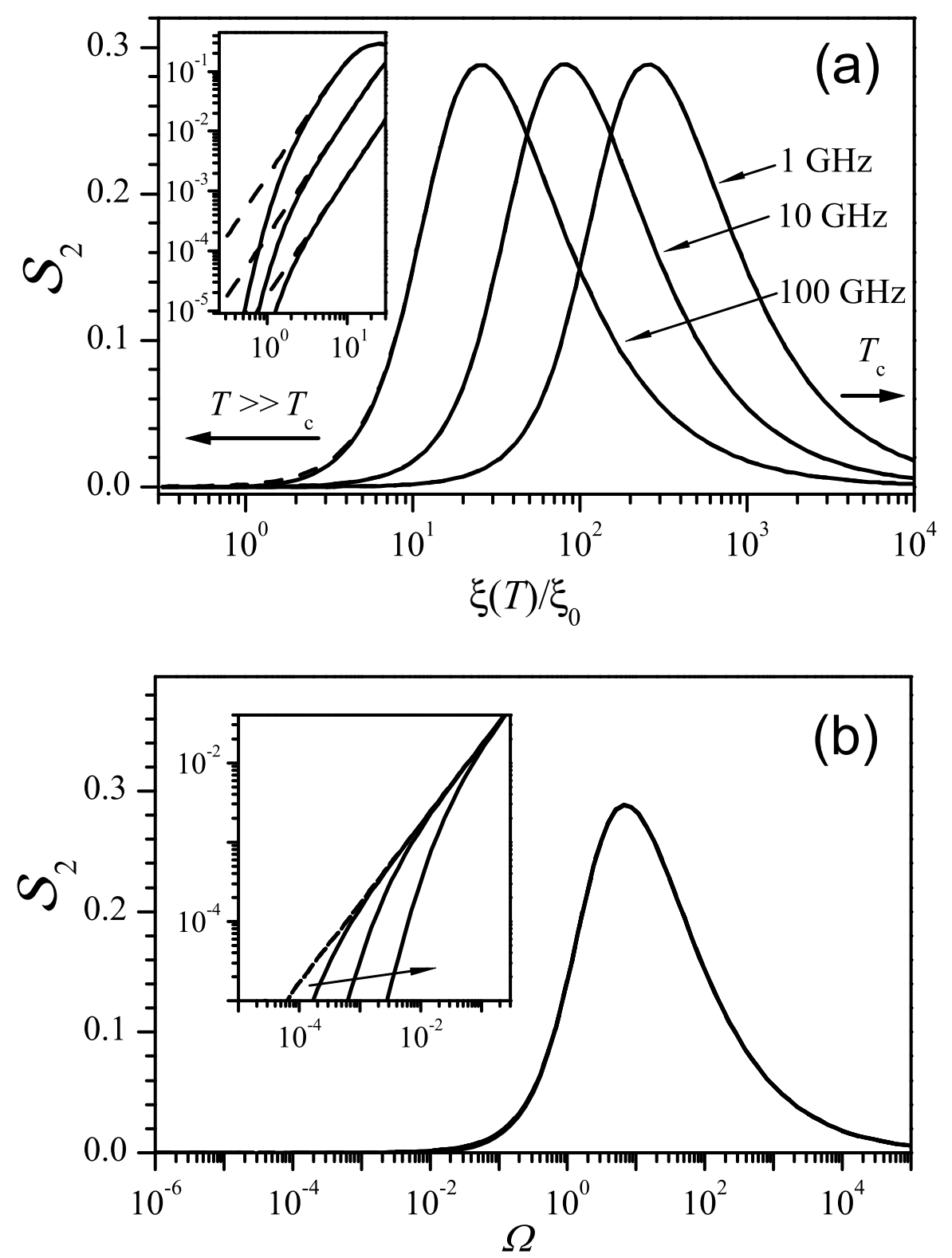

FIG. 2: $\mathcal{S}_{2}$-curves for the $3 D$ isotropic case calculated from Eq. (13) for the same parameters as in Fig. 11 The effects of a finite cutoff are small and can be seen only on logarithmic scales used in the insets. 

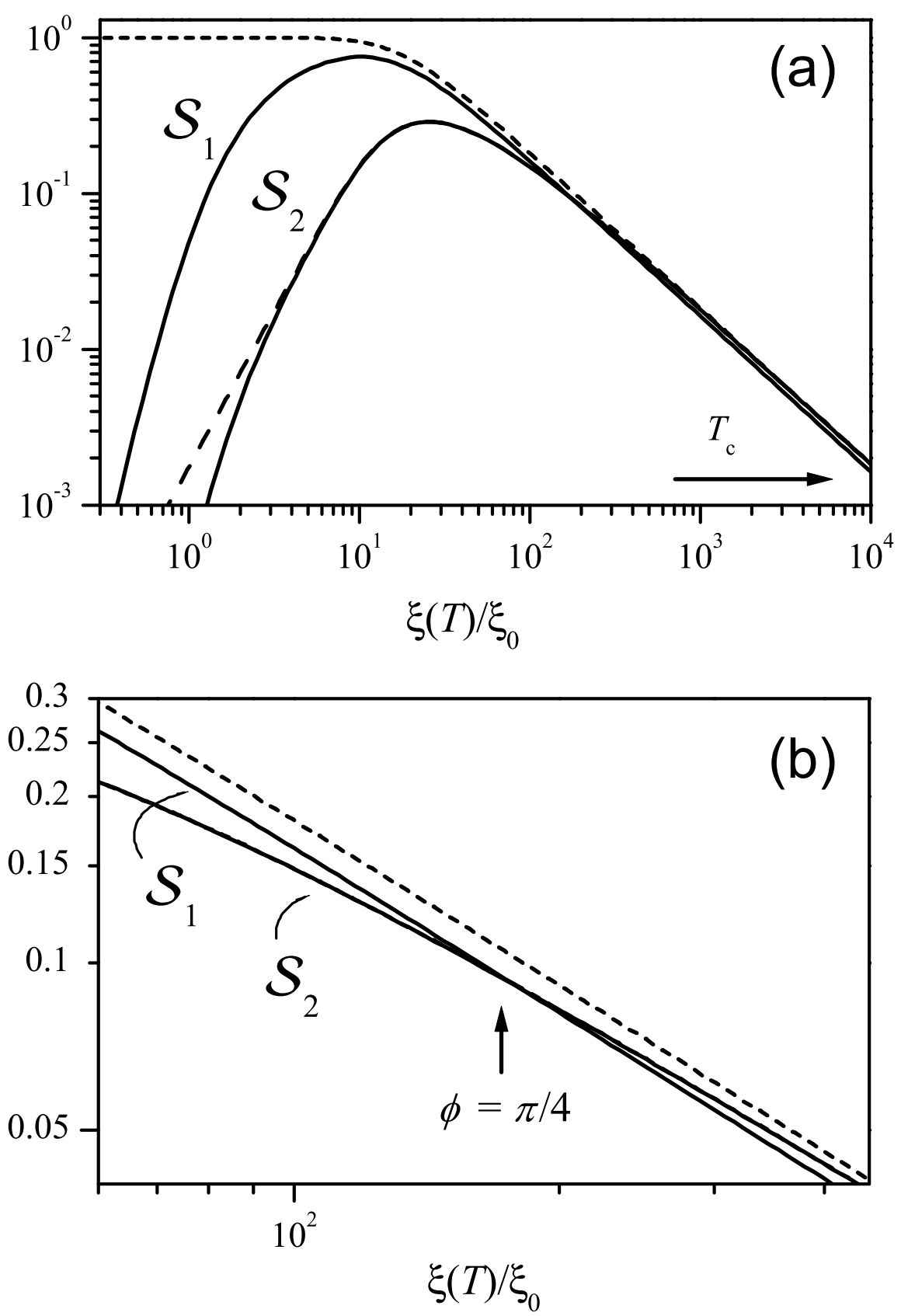

FIG. 3: (a) Asymptotic behavior of the functions $\mathcal{S}_{1}$ and $\mathcal{S}_{2}$ of the $3 D$ isotropic case calculated with the choice $\omega / 2 \pi=100$ $\mathrm{GHz}$ in the limit $T \rightarrow T_{c}$. The dashed lines are the $\mathcal{S}_{1,2}$-curves calculated with no cutoff $(\Lambda=\infty)$, and the full lines include a finite cutoff $(\Lambda=0.5)$. (b) Enlarged section which shows the crossing of $\mathcal{S}_{1}$ and $\mathcal{S}_{2}(\phi=\pi / 4$, see text) at a temperature slightly above $T_{c}$. The two dashed lines are indistinguishable in this temperature range. 

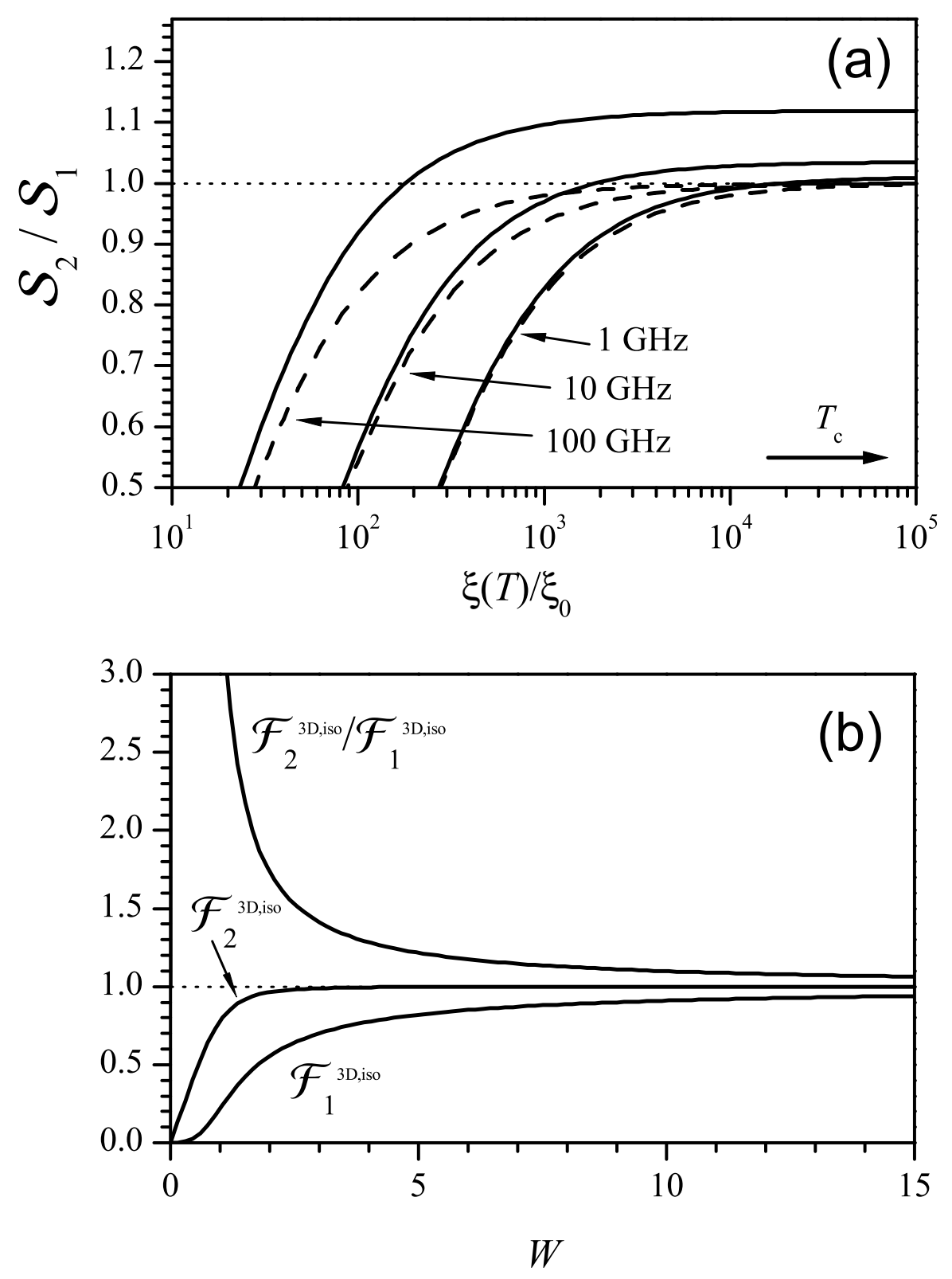

FIG. 4: (a) The ratio $\mathcal{S}_{2} / \mathcal{S}_{1}$ (equal to $\sigma_{2} / \sigma_{1}$ ) for the $3 D$ isotropic case at temperatures approaching $T_{c}$. With no cutoff (dashed lines) the ratio tends to unity for all frequencies. With a finite cutoff $(\Lambda=0.5)$ the ratio equals unity at a temperature slightly above $T_{c}$, dependent on the frequency. In the limit $T \rightarrow T_{c}$ the ratio saturates to a frequency dependent value larger than unity. (b) The ratio $\mathcal{S}_{2} / \mathcal{S}_{1}$ and $\mathcal{F}_{1,2}$ given by Eq. (22). The variable $W$ is defined by Eq. (20). 

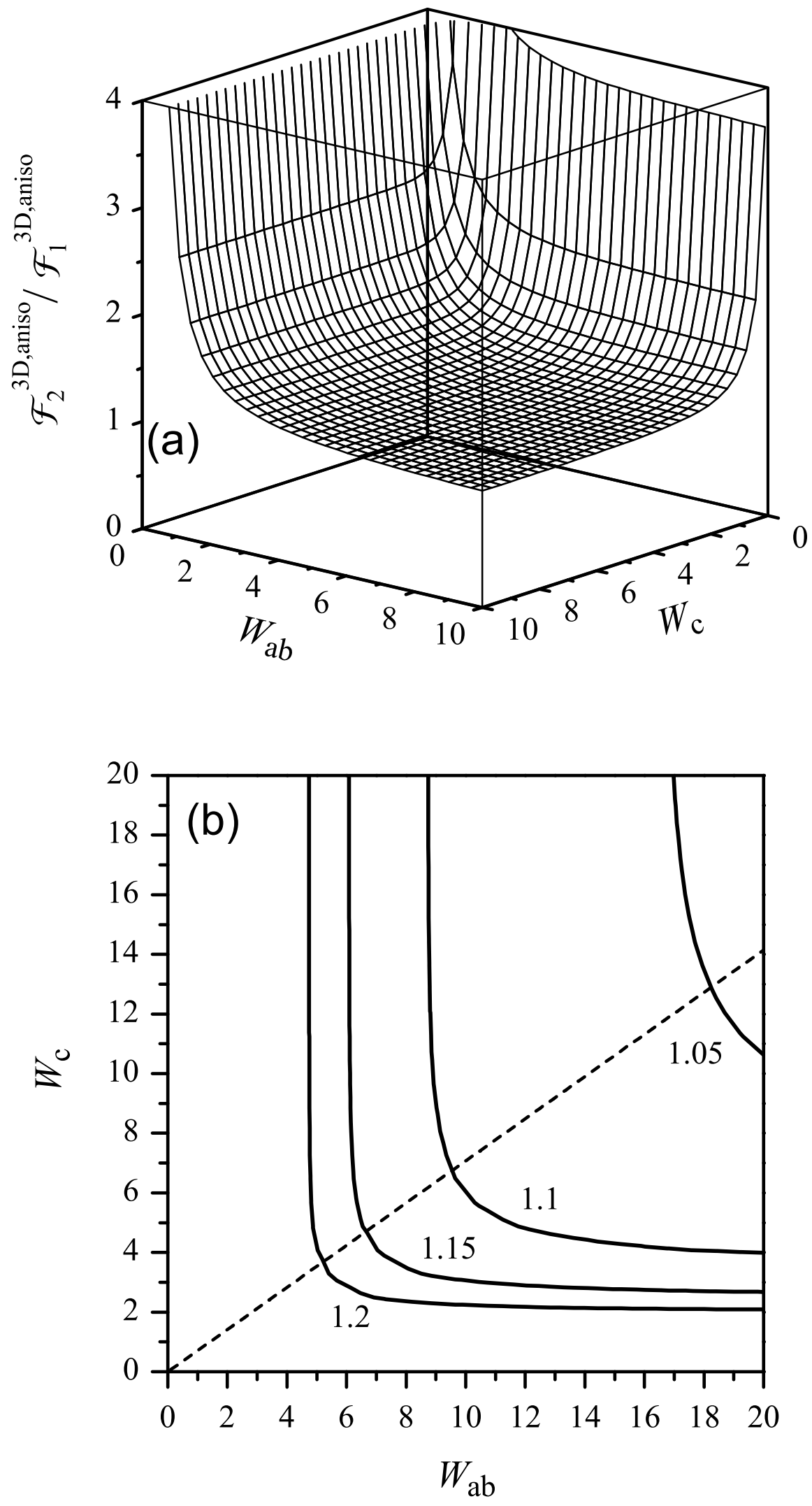

FIG. 5: (a) The ratio $\mathcal{F}_{2} / \mathcal{F}_{1}$ for $3 D$ anisotropic case as a function of $W_{a b}$ and $W_{c}$ (cf. Eq. (52)). (b) Selection of curves for fixed ratios of $\mathcal{F}_{2} / \mathcal{F}_{1}$ indicated by numbers. The dashed line marks the condition $\Lambda_{a b}=\Lambda_{c}\left(W_{a b}=\sqrt{2} W_{c}\right)$. 

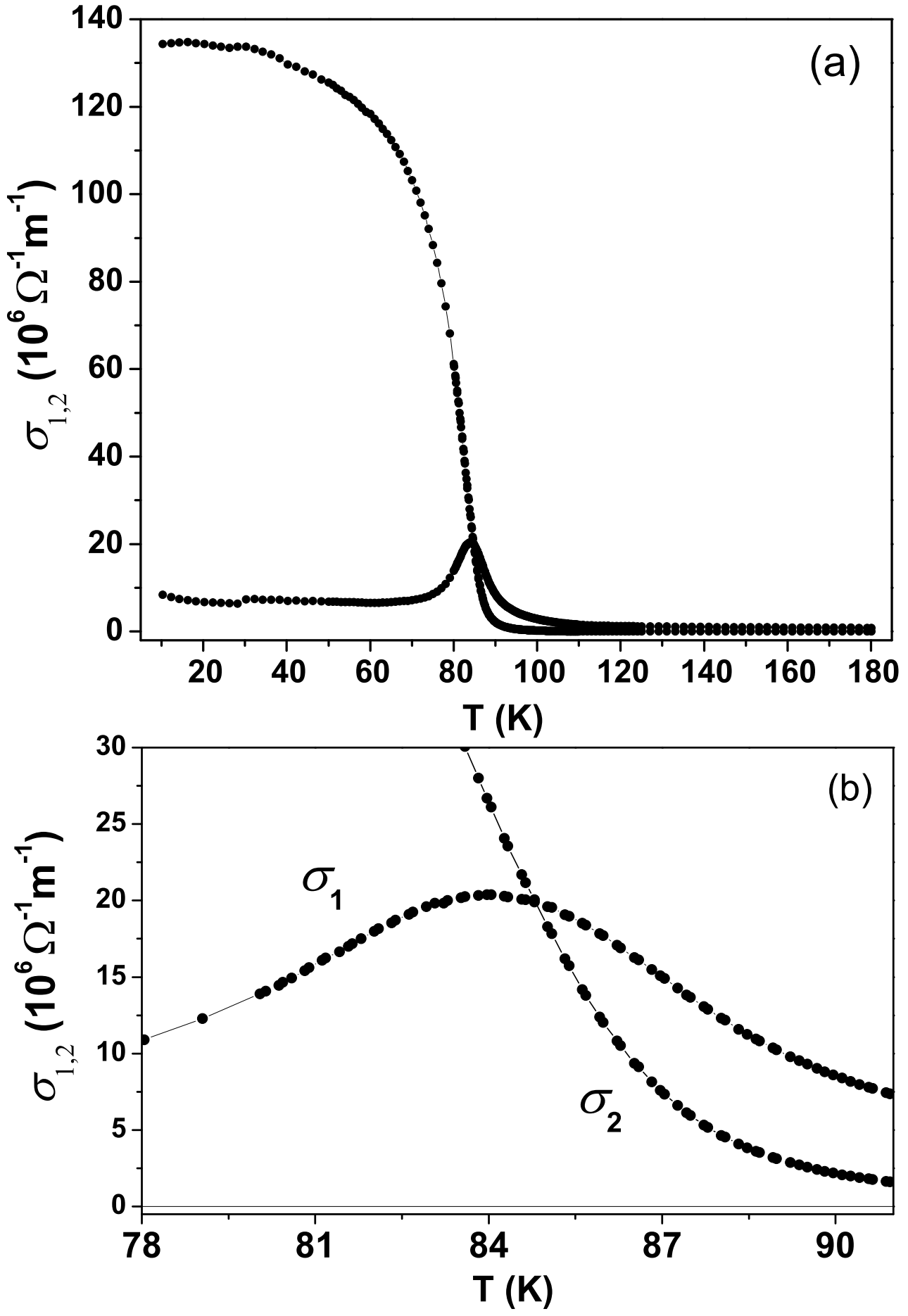

FIG. 6: (a) Experimental complex conductivity in $\mathrm{Bi}_{2} \mathrm{Sr}_{2} \mathrm{Ca}_{2} \mathrm{Cu}_{3} \mathrm{O}_{10-\delta}$ thin film at $9.5 \mathrm{GHz}$. (b) Enlarged section near $\mathrm{T}_{c}$. 

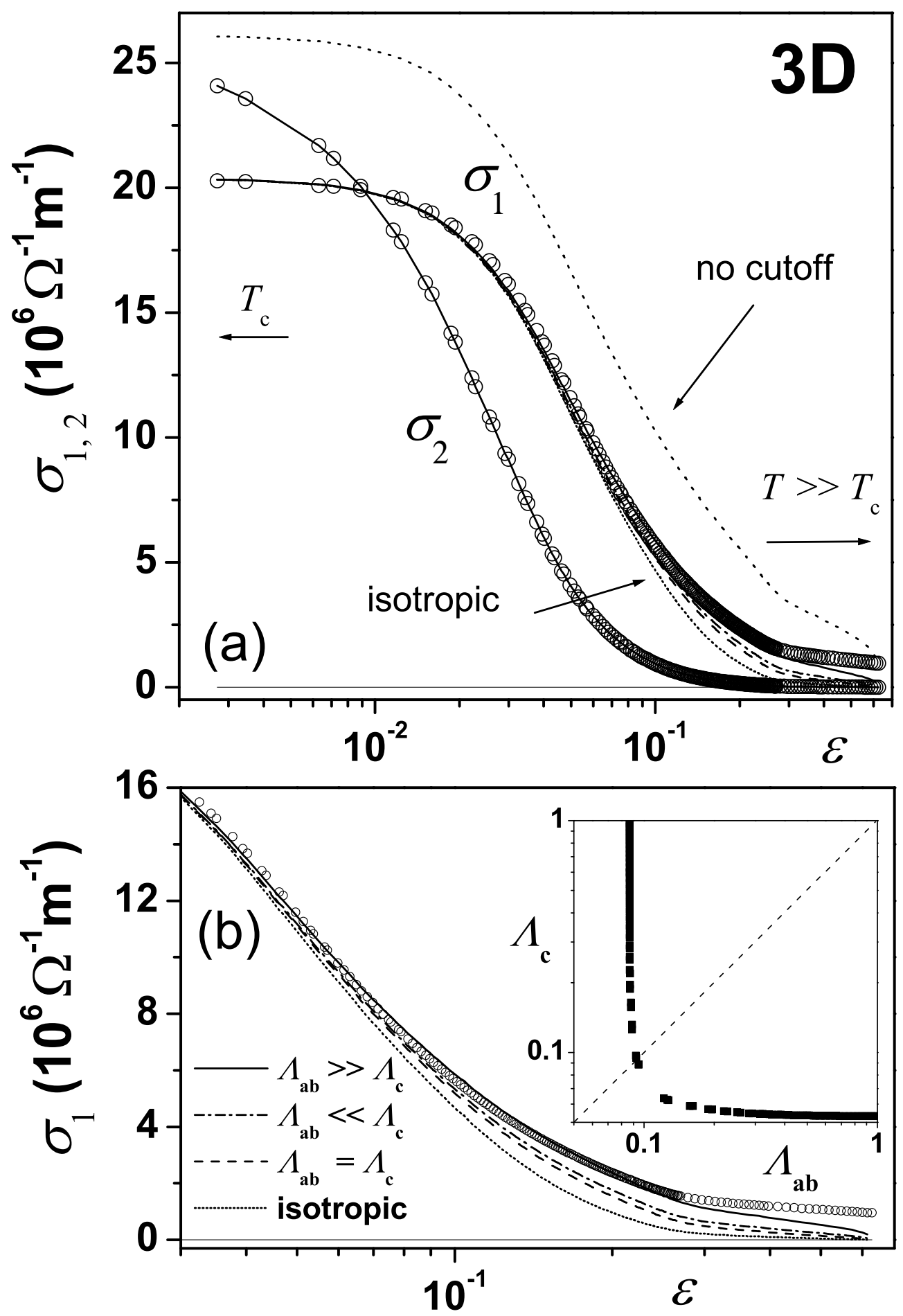

FIG. 7: (a) Experimental data (symbols) of the complex conductivity from Fig. [6] above $T_{c}$ plotted versus the reduced temperature $\epsilon=\left(\ln T / T_{c}\right)$. Various lines are the conductivities calculated in the $3 D$ cases as described in the text. (b) Enlarged view of the high temperature part of the same curves as in (a). The constraining curve for the choices of the cutoff parameters $\Lambda_{a b}$ and $\Lambda_{c}$ resulting from the experimental ratio $\sigma_{2}\left(T_{c}\right) / \sigma_{1}\left(T_{c}\right)=1.28$ at $T_{c}$ is shown in the inset. 

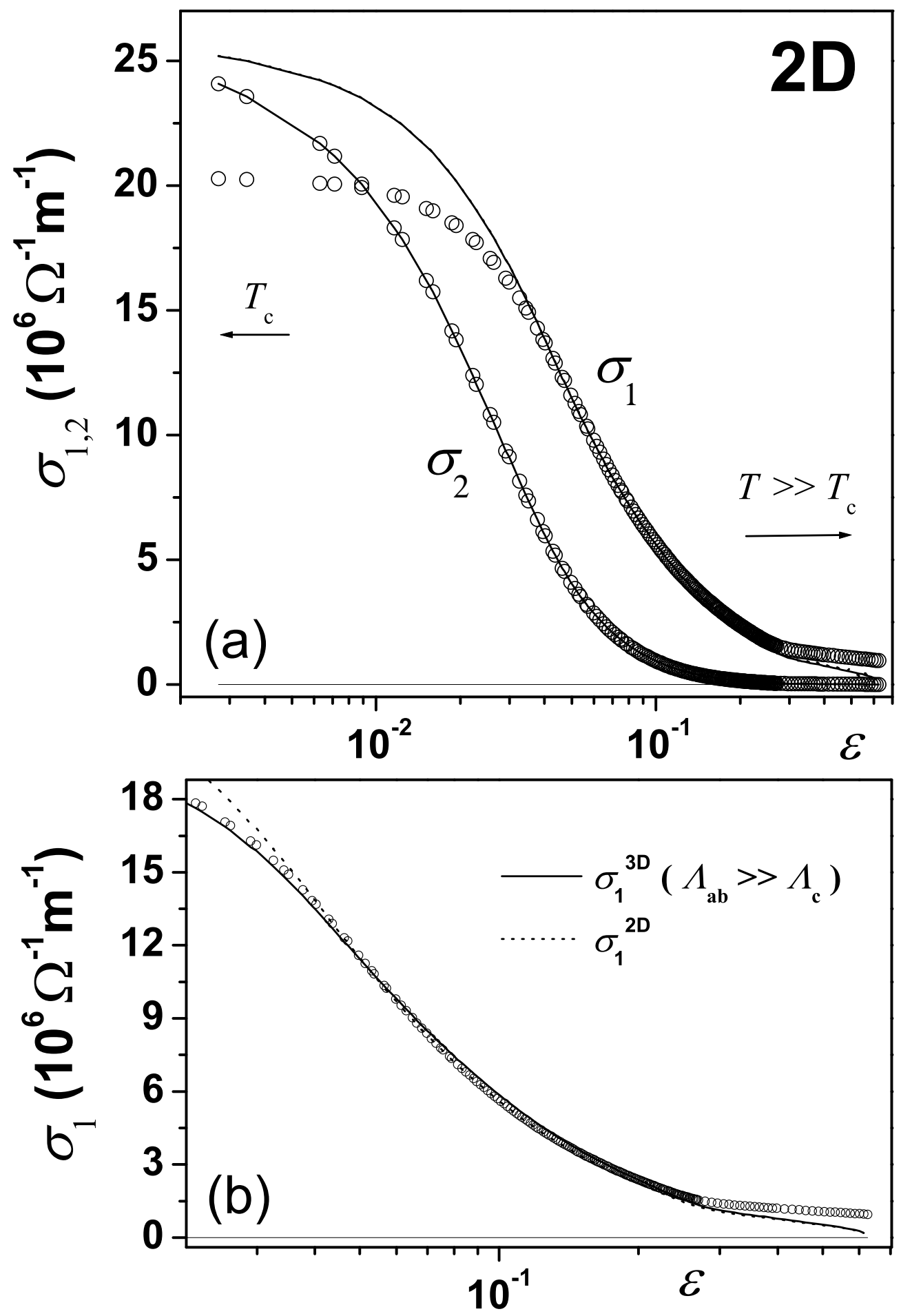

FIG. 8: (a) Experimental data (symbols) as in Fig. 17a) with the calculated $2 D$ curve as described in the text. (b) The superposition of the calculated $2 D$ and $3 D$ curves. 